Department of Policy and Planning Sciences

\author{
Discussion Paper Series
}

No.1355

Multiplicative bias correction for asymmetric

kernel density estimators revisited

by

Gaku IGARASHI and Yoshihide KAKIZAWA

Aug 2018

UNIVERSITY OF TSUKUBA

Tsukuba, Ibaraki 305-8573

JAPAN 


\title{
Multiplicative bias correction for asymmetric kernel density estimators revisited
}

\author{
Gaku IGARASHI ${ }^{1}$ and Yoshihide KAKIZAWA ${ }^{2}$
}

${ }^{1}$ Department of Policy and Planning Sciences, Faculty of Engineering, Information and Systems, University of Tsukuba, 1-1-1 Tennodai, Tsukuba, Ibaraki 305-8573, Japan

${ }^{2}$ Faculty of Economics, Hokkaido University, Nishi 7, Kita 9, Kita-ku, Sapporo 060-0809, Japan

\begin{abstract}
This paper revisits multiplicative bias correction for some asymmetric kernel density estimators (KDEs) when the data is supported on $[0, \infty)^{d}$ or $[0,1]^{d}$. The original method was introduced by Jones et al. (1995) for the standard KDE with symmetric kernel. After Hirukawa (2010) for beta KDE, there have been renewed interests for applications to the asymmetric KDEs. We stress that the variance manipulation must be performed by looking at four terms from the law of total variance/covariance, in which only one term is negligible, while other three terms contribute to the variance formula. It turns out that, even for recently developed asymmetric KDEs, the achievement of the reduced bias is available, at the expense of the constant-factor inflation of the variance. Interestingly, the same factor appears in other bias correction methods.
\end{abstract}

Keywords: multiplicative bias correction; nonparametric density estimation; boundary bias problem; asymmetric kernel.

\section{Introduction}

The kernel density estimator (KDE), introduced by Rosenblatt (1956), is perhaps the most popular in the context of nonparametric density estimation. Several asymptotic results using the location-scale form $K_{h}(\cdot-x)$, with $K_{h}(\cdot)=K(\cdot / h) / h$, where $K$ is a kernel and $h>0$ is a bandwidth, have been well established when the support $\mathcal{S}$ of the underlying density is $\mathbb{R}$. See, e.g., Silverman (1986) and Wand and Jones (1995). However, if $\mathcal{S} \neq \mathbb{R}$, the standard KDE is, in general, inconsistent, due to the bias that is $O(1)$ near the boundary. For this boundary bias problem, various remedies have been discussed in the literature; for example, renormalization, reflection, and generalized jackknifing (Jones (1993)), transformation (Marron and Ruppert (1994)), and advanced reflection (Zhang et al. (1999)).

During recent years, when $\mathcal{S} \neq \mathbb{R}$, there has been a growing interest of the development of the nonparametric density estimation using a certain asymmetric kernel. To the best of our knowledge, Silverman (1986, page 28) first mentioned the possibility of using gamma or log-normal (LN) kernel for the nonnegative data, and Chen (1999) did pioneering studies on beta KDE using a beta kernel for the data from the unit interval. Note that Chen's beta KDE is boundary bias free and nonnegative. Since then, there have been many attempts to suggest a suitable asymmetric kernel $K(\cdot ; x, \beta)$, where

Email: g-igarashi@sk.tsukuba.ac.jp (G. Igarashi), kakizawa@econ.hokudai.ac.jp (Y. Kakizawa).

The first author has been supported by the Japan Society for the Promotion of Science (JSPS); Grant-in-Aid for Young Scientists (B) [Grant Number 17K13714]. The second author has been supported by the JSPS; Grant-in-Aid for Scientific Research (C) [Grant Number 17K00041]. 
$x$ is the location where the density estimation is made, and $\beta>0$ is a smoothing parameter. Given an iid sample $\left\{X_{1}, \ldots, X_{n}\right\}$ from the density $f$ with the support $\mathcal{S}$, we construct an average estimator

$$
\widetilde{f}_{\beta}(x)=\frac{1}{n} \sum_{i=1}^{n} K\left(X_{i} ; x, \beta\right), \quad x \in \mathcal{S},
$$

which is customarily called an asymmetric $\operatorname{KDE}$ when $K(\cdot ; x, \beta)$ is chosen to be supported on $\mathcal{S} \neq \mathbb{R}$. The following points should be distinguished between the classical KDE and the recent asymmetric KDE. Whenever $\mathcal{S} \neq \mathbb{R}$, the support of the asymmetric kernel $K(\cdot ; x, \beta)$ at the location $x \in \mathcal{S}$ under consideration matchs the support $\mathcal{S}$ of the underlying density, whereas any location-scale kernel $K_{h}(\cdot-x)$, at the location $x$ near the boundary, necessarily has a mass outside the support $\mathcal{S}$. Such an asymmetric KDE has often been built within a certain parametric form like (generalized) gamma density, inverse Gaussian (IG) density, reciprocal IG (RIG) density, Birnbaum-Saunders (BS) density, LN density, and so on. See, e.g., Igarashi and Kakizawa (2018a) and references cited therein.

Typically, when the support $\mathcal{S}$ of the density $f$ to be estimated has only one boundary point $\{0\}$ (for the case $\mathcal{S}=[0,1],\{0\}$ should read $\{0,1\}$, of course), there exist constants ${ }^{[1]} q, r, r^{\prime}>0 ; q \geq r \geq r^{\prime}$ and functions $\gamma_{1}(\cdot ; f)$ and $\delta(\cdot)$, independent of $\beta$, such that, for all sufficiently small $\beta>0$,

$$
\begin{aligned}
& \operatorname{Bias}\left[\widetilde{f}_{\beta}(x)\right]=\beta^{q} \gamma_{1}(x ; f)+o\left(\beta^{q}\right), \\
& \operatorname{Var}\left[\widetilde{f}_{\beta}(x)\right]=\left\{\begin{array}{l}
n^{-1} \beta^{-r^{\prime}} \delta(x) f(x)+o\left(n^{-1} \beta^{-r^{\prime}}\right) \quad \text { for } x \in \mathcal{S} \backslash\{0\}, \\
n^{-1} \beta^{-r} \delta(0) f(0)+o\left(n^{-1} \beta^{-r}\right) \quad \text { for } x=0 .
\end{array}\right.
\end{aligned}
$$

Then, assuming that $\beta=\beta_{n} \searrow 0$ and $n \beta^{r} \rightarrow \infty$ (in this case, $n \beta^{r^{\prime}} \rightarrow \infty$ ) as $n \rightarrow \infty$, the leading term of the mean squared error $M S E\left[\widetilde{f}_{\beta}(x)\right]$ (in short, asymptotic MSE (AMSE)) is given by

$$
A M S E\left[\widetilde{f}_{\beta}(x)\right]=\left\{\begin{array}{l}
\beta^{2 q} \gamma_{1}^{2}(x ; f)+n^{-1} \beta^{-r^{\prime}} \delta(x) f(x) \quad \text { for } x \in \mathcal{S} \backslash\{0\}, \\
\beta^{2 q} \gamma_{1}^{2}(0 ; f)+n^{-1} \beta^{-r} \delta(0) f(0) \quad \text { for } x=0
\end{array}\right.
$$

(i.e., the asymmetric KDE is pointwise consistent). Choosing

$$
\beta^{o p t}(x)= \begin{cases}{\left[\frac{r^{\prime} \delta(x) f(x)}{2 q \gamma_{1}^{2}(x ; f)} n^{-1}\right]^{1 /\left(2 q+r^{\prime}\right)}} & \text { for } x \in \mathcal{S} \backslash\{0\}, \\ {\left[\frac{r \delta(0) f(0)}{2 q \gamma_{1}^{2}(0 ; f)} n^{-1}\right]^{1 /(2 q+r)}} & \text { for } x=0,\end{cases}
$$

we have the optimal AMSE;

$$
A M S E^{o p t}\left[\widetilde{f}_{\beta}(x)\right]= \begin{cases}\frac{2 q+r^{\prime}}{r^{\prime}}\left\{\gamma_{1}^{2}(x ; f)\right\}^{r^{\prime} /\left(2 q+r^{\prime}\right)}\left[\frac{r^{\prime}}{2 q} \delta(x) f(x) n^{-1}\right]^{2 q /\left(2 q+r^{\prime}\right)} & \text { for } x \in \mathcal{S} \backslash\{0\}, \\ \frac{2 q+r}{r}\left\{\gamma_{1}^{2}(0 ; f)\right\}^{r /(2 q+r)}\left[\frac{r}{2 q} \delta(0) f(0) n^{-1}\right]^{2 q /(2 q+r)} & \text { for } x=0 .\end{cases}
$$

\footnotetext{
${ }^{[1]}$ The particular case of $q=r=1$ and $r^{\prime}=1 / 2$ is important, in accordance with the pioneering work on the gamma KDE (Chen (2000)). However, it would not be guaranteed generally, except that one index " $r$ " (say) could be assumed to be 1 , without loss of generality.
} 


\subsection{Additive bias reduction}

Improving the performance (4) is an important topic even when $\mathcal{S} \neq \mathbb{R}$. There have been recent renewed interests on bias reductions for the asymmetric KDE (1). Suppose that, in addition to (2),

$$
\operatorname{Bias}\left[\widetilde{f}_{\beta}(x)\right]=\sum_{j=1}^{2} \beta^{j q} \gamma_{j}(x ; f)+o\left(\beta^{2 q}\right),
$$

for some function $\gamma_{2}(\cdot ; f)$, independent of $\beta$. In this case, the easiest way to remove the $O\left(\beta^{q}\right)$ bias is perhaps an additive bias reduction due to Schucany and Sommers (1977). That is, for each constant $a \in(0,1)$, the estimator, defined by

$$
\widetilde{f}_{\beta, S S_{a}}(x)=\frac{1}{1-a^{q}} \widetilde{f}_{\beta}(x)-\frac{a^{q}}{1-a^{q}} \widetilde{f}_{\beta / a}(x), \quad x \in \mathcal{S} \quad \text { (we call the SS-type) }
$$

has the asymptotic bias, $\operatorname{Bias}\left[\tilde{f}_{\beta, S S_{a}}(x)\right]=-\left(\beta^{2 q} / a^{q}\right) \gamma_{2}(x ; f)+o\left(\beta^{2 q}\right)$. This technique was originally applied to the standard KDE, and recently to the asymmetric KDEs. See Leblanc (2010), Igarashi and Kakizawa (2014a, 2015, 2018a), and Igarashi (2016a) for the data supported on $\mathcal{S}=[0, \infty)$ or $[0,1]$. Furthermore, as discussed in Jones and Foster (1993, Example 2.1) for the standard KDE, and Igarashi and Kakizawa (2015, 2018b) and Igarashi (2016a) for the asymmetric KDEs, if the estimator (1) is differentiable with respect to $\beta$, the limiting estimator, defined by

$$
\lim _{a \rightarrow 1} \tilde{f}_{\beta, S S_{a}}(x)=\widetilde{f}_{\beta}(x)-\frac{\beta}{q} \frac{\partial}{\partial \beta} \widetilde{f}_{\beta}(x)=\widetilde{f}_{\beta, S S_{1}}(x) \quad(\text { say }), \quad x \in \mathcal{S},
$$

will have the asymptotic bias, $\operatorname{Bias}\left[\widetilde{f}_{\beta, S S_{1}}(x)\right]=-\beta^{2 q} \gamma_{2}(x ; f)+o\left(\beta^{2 q}\right)$. Here, the SS-type estimator is written as the form (1), i.e., $\widetilde{f}_{\beta, S S_{a}}(x)=n^{-1} \sum_{i=1}^{n} K_{S S_{a}}\left(X_{i} ; x, \beta\right), x \in \mathcal{S}$, where

$$
K_{S S_{a}}(s ; x, \beta)= \begin{cases}\frac{1}{1-a^{q}} K(s ; x, \beta)-\frac{a^{q}}{1-a^{q}} K(s ; x, \beta / a), & a \in(0,1), \\ K(s ; x, \beta)-\frac{\beta}{q} \frac{\partial}{\partial \beta} K(s ; x, \beta), & a=1,\end{cases}
$$

so that the resulting asymmetric kernel will be interpreted as "a higher order asymmetric kernel" derived from a given asymmetric kernel $K(\cdot ; x, \beta)$ only ${ }^{[2]}$. Its asymptotic variance and AMSE are found in Igarashi and Kakizawa (2015, 2018a, 2018b) and Igarashi (2016a) for the data supported on $\mathcal{S}=[0, \infty)$ or $[0,1]$ (if $a \in(0,1)$, an additional task is to approximate $\operatorname{Cov}\left[\tilde{f}_{\beta}(x), \widetilde{f}_{\beta / a}(x)\right]$ ). Compared to the (uncorrected) estimator $\widetilde{f}_{\beta}(x)$, the order of $V\left[\widetilde{f}_{\beta, S S_{a}}(x)\right]$ remains unchanged, but the coefficient of the leading term of $V\left[\widetilde{f}_{\beta, S S_{a}}(x)\right]$ increases with the factor

$$
\lambda(a)=\frac{1}{(1-a)^{2}}\left\{1-2 a\left(\frac{2 a}{a+1}\right)^{1 / 2}+a^{5 / 2}\right\},
$$

where $\lambda$ is increasing for $a \in(0,1) ; \lim _{a \rightarrow 0} \lambda(a)=1$ and $\lim _{a \rightarrow 1} \lambda(a)=27 / 16$. Interestingly, the factor $\lambda(a)$ appeared in Wand and Schucany (1990) for the (standard) Gaussian $\operatorname{KDE}(\mathcal{S}=\mathbb{R})$. It should be remarked that, although the $\mathrm{SS}_{a}$-type, for each $a \in(0,1]$, loses the nonnegativity by construction, the positive part estimator $\widetilde{f}_{\beta, S S_{a}}^{+}(x)=\max \left\{0, \widetilde{f}_{\beta, S S_{a}}(x)\right\}$ not only keeps the nonnegativity, but also improves the performance in the sense that $M S E\left[\widetilde{f}_{\beta, S S_{a}}^{+}(x)\right] \leq M S E\left[\widetilde{f}_{\beta, S S_{a}}(x)\right]$.

\footnotetext{
${ }^{[2]}$ For the standard KDE using the location-scale form $K_{[2]}((\cdot-x) / h) / h$ as the kernel, Jones and Foster (1993) considered an enormous variety of higher order kernels from a given 2 nd order kernel $K_{[2]}$ only, by generalized jackknifing.
} 


\subsection{Nonnegative bias reductions}

There have been other bias reduction methods due to Terrell and Scott (1980) and Jones and Foster (1993), that are guaranteed to be nonnegative. Technically, introducing a parameter $\epsilon \searrow 0$ to avoid the division by zero, two nonnegative bias-corrected estimators (we call the TS-type and JF-type) are, respectively, defined by, for each constant $a \in(0,1)$,

$$
\widetilde{f}_{\beta, T S_{a}}(x)=\frac{\left\{\widetilde{f}_{\beta}(x)+\epsilon\right\}^{1 /\left(1-a^{q}\right)}}{\left\{\widetilde{f}_{\beta / a}(x)+\epsilon / a^{q}\right\}^{q^{q} /\left(1-a^{q}\right)}}, \quad \widetilde{f}_{\beta, J F_{a}}(x)=\left\{\widetilde{f}_{\beta}(x)+\epsilon\right\} \exp \left\{\frac{\widetilde{f}_{\beta, S S_{a}}(x)}{\widetilde{f}_{\beta}(x)+\epsilon}-1\right\}, \quad x \in \mathcal{S}
$$

(Terrell and Scott (1980) and Jones and Foster (1993) originally used $\epsilon=0$ for the standard KDE $(\mathcal{S}=\mathbb{R}))$. By construction, we can see that, if $\epsilon$ is independent of $a$, then,

$$
\lim _{a \rightarrow 1} \widetilde{f}_{\beta, T S_{a}}(x)=\lim _{a \rightarrow 1} \widetilde{f}_{\beta, J F_{a}}(x)=\left\{\widetilde{f}_{\beta}(x)+\epsilon\right\} \exp \left\{\frac{\widetilde{f}_{\beta, S S_{1}}(x)}{\widetilde{f}_{\beta}(x)+\epsilon}-1\right\}, \quad x \in \mathcal{S}
$$

(hence, the TS-type is linked with the JF-type; $\widetilde{f}_{\beta, T S_{1}}(x)=\widetilde{f}_{\beta, J F_{1}}(x)$ ). As demonstrated in Igarashi (2016a) and Igarashi and Kakizawa (2018a, 2018b) (note that a careful analysis of the remainder term was carried out there), the TS-type and JF-type have the stochastic expansions

$$
\widetilde{f}_{\beta, \# a}(x) \approx \widetilde{f}_{\beta, S S_{a}}(x)+\frac{\left\{\widetilde{f}_{\beta}(x)-\widetilde{f}_{\beta, S S_{a}}(x)+\epsilon\right\}^{2}}{2 a^{\chi_{\{\#=T S\}}} f(x)}, \quad \#=T S, J F, \quad \text { if } f(x)>0,
$$

which help understanding of asymptotic properties of the $\mathrm{TS}_{a}$-type and $\mathrm{JF}_{a}$-type similar to those of the $\mathrm{SS}_{a}$-type, except for an additional bias term coming from $E\left[\left\{\widetilde{f}_{\beta}(x)-\widetilde{f}_{\beta, S S_{a}}(x)+\epsilon\right\}^{2} / f(x)\right] /\left(2 a^{\chi_{\{\#=T S\}}}\right)$. Consequently, the $\mathrm{TS}_{a}$-type and $\mathrm{JF}_{a}$-type bias reductions work even for the asymmetric KDEs, at expense of the constant-factor inflation; $\lambda(a)$ of the variance, as in the $\mathrm{SS}_{a}$-type bias reduction.

On the other hand, in the seminal paper, Jones et al. (1995) developed another multiplicative (hence, nonnegative) bias reduction method (we call the JLN-type), focusing on the standard KDE $(\mathcal{S}=\mathbb{R})$. A possible application to asymmetric KDEs was mentioned in Hagmann and Scaillet (2007). Hirukawa (2010, correction 2016) showed that the asymptotic variance of the JLN-type bias-corrected beta $\mathrm{KDE}$ is equivalent to that of the (uncorrected) beta $\mathrm{KDE}$, despite the variance inflation $\lambda(a)$ for the $\mathrm{TS}_{a}$-type bias-corrected beta $\mathrm{KDE}$ (the $\mathrm{TS}_{1}$-type or $\mathrm{JF}_{a}$-type bias-corrected beta $\mathrm{KDE}$ was additionally studied by Igarashi (2016a)). However ${ }^{[3]}$, as will be revisited in this paper, it turns out that Hirukawa's asymptotic variance miss two terms, and, to make matters worse, Hirukawa's original incorrect proof may lead to similar incorrect conclusions in his companion papers (Hirukawa and Sakudo (2014, 2015)) and subsequent papers (Funke and Kawka (2015), Zougab and Adjabi (2016), and Zougab et al. (2018)). To be exact, as Jones et al. (1995) did for the standard $\operatorname{KDE}(\mathcal{S}=\mathbb{R})$, we need, for the variance manipulation, to look at four terms from the law of total variance/covariance, in which only one term is negligible, while other three terms contribute to the final result (i.e., the aforementioned authors's asymptotic variances would be incorrectly asserted in common).

\footnotetext{
${ }^{[3]}$ The first author, in his master thesis (March, 2012; in Japanese), realized that the asymptotic variances of the JLN-type bias-corrected beta/gamma/Bernstein KDEs have the inflation factor $\lambda(1 / 2)$. However, his proof at that time was formal without a rigorous analysis of the remainder term of the stochastic expansion.
} 


\subsection{Overview of the paper}

The contribution of this paper is three fold. First, we are changing the original JLN-type definition involved the division by $\widetilde{f}_{\beta}\left(X_{i}\right), i=1, \ldots, n$, as follows: In line with Jones et al. (1995) (they originally used $\epsilon=0$ for the standard $\operatorname{KDE}(\mathcal{S}=\mathbb{R}))$, we define the multiplicative bias-corrected estimator by

$$
\widetilde{f}_{\beta, J L N}(x)=\left\{\widetilde{f}_{\beta}(x)+\epsilon\right\} \frac{1}{n} \sum_{i=1}^{n} \frac{K\left(X_{i} ; x, \beta\right)}{\widetilde{f}_{\beta}\left(X_{i}\right)+\epsilon}, \quad x \in \mathcal{S},
$$

where the introduction of $\epsilon \searrow 0$ avoids dividing by zero. By construction, this estimator keeps the nonnegativity. Second, we are carefully examining the asymptotic negligibility of the remainder term of the stochastic expansion, in the spirit of Chen et al. (2009) (see Igarashi and Kakizawa (2018a)). The basic tools are the Rosenthal and Bennett inequalities of the absolute moment and tail probability of the sum of zero-mean independent random variables (and their conditional variants). Third, revisiting the variance manipulation, it is shown that the JLN-type bias reduction, even when it is applied to the asymmetric KDEs, works, at expense of the constant-factor inflation of the variance, whose factor is given by $\lambda(1 / 2)=4-4 \sqrt{2} / \sqrt{3}+1 / \sqrt{2} \approx 1.441$, at least, for three specific asymmetric KDEs suggested in Igarashi and Kakizawa $(2015,2018 \mathrm{a})$ and Igarashi $(2016 \mathrm{~b})(\mathcal{S}=[0, \infty))$, or the beta $\operatorname{KDE}(\mathcal{S}=[0,1])$. Interestingly, the same factor $\lambda(1 / 2)$ appeared in the $\mathrm{SS}_{1 / 2}$-type, $\mathrm{TS}_{1 / 2}$-type, and $\mathrm{JF}_{1 / 2}$-type. It is worth noting that the ratio of the integral $\int_{-\infty}^{\infty}\{2 \phi(u)-(\phi * \phi)(u)\}^{2} d u$ relative to the integral $\int_{-\infty}^{\infty} \phi^{2}(u) d u$ is equal to $\lambda(1 / 2)$, where $\phi$ is Gaussian density and $*$ is a convolution operator.

The rest of the paper is organized as follows. After presenting our assumptions, Section 2 gives the main results for the JLN-type bias-corrected estimator (6). Section 3 is devoted to the special cases of three different families of asymmetric KDEs for the nonnegative data. Section 4 describes extension to $d$-variate density estimation by using the product kernel method. Section 5 discusses the product-type beta $\mathrm{KDE}$ for the data supported on $[0,1]^{d}$. In Section 6 , we present results from simulation studies. The proofs are given in the Appendix; some technical details are deferred without further reference to an supplemental issue: Supplemental appendix to "Multiplicative bias correction for asymmetric kernel density estimators revisited", Faculty of Economics, Hokkaido University, Discussion Paper Series A: No. 2018-328.

Notation. The dependency on the sample size $n$ is suppressed (e.g., the smoothing parameter is denoted by $\beta$, instead of $\beta_{n}$ ), but, unless otherwise stated, the limits will be taken as $n \rightarrow \infty$. We use the notation $\|h\|_{\mathcal{S}}=\sup _{x \in \mathcal{S}}|h(x)|$ for any bounded function $h$ on $\mathcal{S}$. As usual, we also denote by $h^{(j)}(x)=(d / d x)^{j} h(x)$ the $j$ th derivative of $h$ (if it exists), and write $h^{(0)}(x)=h(x)$.

\section{JLN-type bias correction}

In what follows, we assume that $\mathcal{S}=[0, \infty)$. Let $\mathcal{X}_{n}=\left\{X_{1}, \ldots, X_{n}\right\}$ be a random sample of size $n$, drawn from a univariate unknown density $f$ with the support $\mathcal{S}$. The goal of this section is to study the JLN-type bias-corrected $\operatorname{KDE}(6)$. We formulate our asymptotic results at given point $x_{0} \in\left[0, c_{U}\right]$, where $c_{U}>0$ is fixed but arbitrary. 
For simplicity, the $j$ th moment around $x(\in \mathcal{S})$ of $K(\cdot ; x, \beta)$ is denoted by

$$
\widetilde{\mu}_{j}(K(\cdot ; x, \beta))=\int_{\mathcal{S}}(t-x)^{j} K(t ; x, \beta) d t \quad \text { (if it exists). }
$$

\subsection{Assumptions}

We make the following assumptions (although, as in Introduction, we can formulate the assumptions indexed by $q, r, r^{\prime}>0 ; q \geq r \geq r^{\prime}$, we here focus on the particular case $q=r=1$ and $r^{\prime}=1 / 2$, in accordance with the gamma KDE (Chen (2000))):

A1. There exists a density $p_{K}(\cdot ; \cdot)$, such that

1. $K(t ; x, \beta)=p_{K}(t / \beta ; x / \beta) / \beta$ for any $t, x \in \mathcal{S}$, where the functional form of $p_{K}$ is independent of $\beta$ and $x$ (this implies that $K(\cdot ; x, \beta)$ is nonnegative and satisfies $\widetilde{\mu}_{0}(K(\cdot ; x, \beta)) \equiv 1$, where $\widetilde{\mu}_{j}(K(\cdot ; x, \beta))=\beta^{j} \int_{\mathcal{S}}(u-x / \beta)^{j} p_{K}(u ; x / \beta) d u$ for $j \in \mathbb{N}$ (if it exists)),

2. the $j$ th raw moment $\mu_{j}^{\prime}(y)=\int_{\mathcal{S}} u^{j} p_{K}(u ; y) d u$ exists for any $y \in \mathcal{S}$, having the polynomial grawth of degree $j$, i.e., $\sup _{y \in \mathcal{S}}\left\{\mu_{j}^{\prime}(y) /(1+y)^{j}\right\}<\infty$, for $j=1,2,3,4,6$, and

3. for any $y \in \mathcal{S}, \mu_{1}^{\prime}(y)=y+\zeta_{1,1}$ for some constant $\zeta_{1,1}$, independent of $y$ (in other words, for any $x \in \mathcal{S}, K(\cdot ; x, \beta)$ satisfies $\left.\widetilde{\mu}_{1}(K(\cdot ; x, \beta))=\beta \zeta_{1,1}\right)$; in this case, the constant $\zeta_{1,1}$ is necessarily equal to $\mu_{1}^{\prime}(0)>0$.

A2. (i) When $x / \beta \rightarrow \infty$ (it holds at least for any fixed bounded $x>0$ ), the $j$ th moments around $x$; $\widetilde{\mu}_{j}(K(\cdot ; x, \beta)), j=2,3,4,6$, admit the asymptotic expansions

$$
\widetilde{\mu}_{j}(K(\cdot ; x, \beta))=\left\{\begin{aligned}
\beta \zeta_{1,2} x+\beta^{2} \zeta_{2,2}+O\left(\beta^{3} x^{-1}\right), & j=2, \\
\beta^{2} \zeta_{2,3} x+O\left(\beta^{3}\right), & j=3, \\
\beta^{2} \zeta_{2,4} x^{2}+O\left(\beta^{3} x\right), & j=4, \\
O\left(\beta^{3} x^{3}\right), & j=6
\end{aligned}\right.
$$

for some constants $\zeta_{1,2}, \zeta_{2,2}, \zeta_{2,3}, \zeta_{2,4}\left(\zeta_{1,2}, \zeta_{2,4}>0\right)$, independent of $\beta$ and $x$. More precisely, the remainder terms $r_{2, \beta}(x)=O\left(\beta^{3} x^{-1}\right), r_{3, \beta}(x)=O\left(\beta^{3}\right), r_{4, \beta}(x)=O\left(\beta^{3} x\right)$, and $r_{6, \beta}(x)=O\left(\beta^{3} x^{3}\right)$ for $j=2,3,4,6$ are estimated, as follows: Given constants $\eta \in[0,1)$ and $c_{L}>0$, for all sufficiently small $\beta>0, x \geq c_{L} \beta^{\eta}$ implies that $\left|r_{2, \beta}(x)\right| \leq M_{2} \beta^{3} /(x+\beta),\left|r_{3, \beta}(x)\right| \leq M_{3} \beta^{3}$, $\left|r_{4, \beta}(x)\right| \leq M_{4} \beta^{3}(x+\beta)$, and $\left|r_{6, \beta}(x)\right| \leq M_{6} \beta^{3}(x+\beta)^{3}$ for some constants $M_{2}, M_{3}, M_{4}, M_{6}>0$, independent of $\beta$ and $x$.

(ii) The following uniform/nonuniform bounds hold:

1. $\sup _{x \in \mathcal{S}} \sup _{s \in \mathcal{S}} K(s ; x, \beta) \leq C_{K} \beta^{-1}$ for some constant $C_{K}>0$, independent of $\beta$.

2. whenever $x>0, \sup _{s \in \mathcal{S}} K(s ; x, \beta) \leq C_{K}^{\prime}(\beta x)^{-1 / 2}$ for some constant $C_{K}^{\prime}>0$, independent of $\beta$ and $x$. 
(iii) Given constants $\eta \in[0,1)$ and $c_{L}>0$, for all sufficiently small $\beta>0, x \geq c_{L} \beta^{\eta}$ implies that

$$
\left|\int_{\mathcal{S}} K^{2}(s ; x, \beta) d s-\frac{1}{2 \sqrt{\pi \beta x}}\right| \leq \frac{M}{\sqrt{\beta x}}\left(\frac{\beta}{x+\beta}\right)
$$

for some constant $M>0$, independent of $\beta$ and $x$.

(iv) Given constants $\eta \in[0,1 / 4)$ and $0<c_{L}<c_{U}$, for all sufficiently small $\beta>0$, the following approximations hold for $x \in\left[c_{L} \beta^{\eta}, c_{U}\right]$ :

$$
\begin{aligned}
\int_{\mathcal{S}} K(t ; x, \beta) \int_{\mathcal{S}} K(s ; x, \beta) K(s ; t, \beta) d s d t & =\frac{1}{\sqrt{6 \pi \beta x}}+o\left((\beta x)^{-1 / 2}\right) \\
\int_{\mathcal{S}} \int_{\mathcal{S}}\left\{\prod_{j=1}^{2} K\left(t_{j} ; x, \beta\right)\right\} \int_{\mathcal{S}}\left\{\prod_{j=1}^{2} K\left(s ; t_{j}, \beta\right)\right\} d s d t_{1} d t_{2} & =\frac{1}{2 \sqrt{2 \pi \beta x}}+o\left((\beta x)^{-1 / 2}\right) .
\end{aligned}
$$

A3. (i) $f$ is four times continuously differentiable on $\mathcal{S}$, with $\sum_{j=0}^{4}\left\|f^{(j)}\right\|_{\mathcal{S}}<\infty$.

(ii) There exist constants $\eta_{4} \in(0,1]$ and $L_{4}>0$, such that $\left|f^{(4)}(y)-f^{(4)}(z)\right| \leq L_{4}|y-z|^{\eta_{4}}$ for any $y, z \in \mathcal{S}$.

A4. (i) There exists a constant $R\left(>c_{U}\right)$, such that, for some $\bar{\ell} \in \mathbb{N}$,

1. $\inf _{0 \leq x \leq R} f(x)>0$, and

2. for all sufficiently small $\beta>0, \sup _{x \in\left[0, c_{U}\right]} \int_{R}^{\infty}\left(1+s^{2}\right) K(s ; x, \beta) / f^{\bar{\ell}}(s) d s$ is smaller than any positive power of $\beta$.

(ii) There exists a constant $\delta \in(0,1)$, such that, given constants $\eta \in[0,1)$ and $0<c_{L}<c_{U}$, for all sufficiently small $\beta>0, \sup _{x \in\left[c_{L} \beta^{\eta}, c_{U}\right]} \int_{0}^{\delta x} K(s ; x, \beta) d s$ is smaller than any positive power of $\beta$.

A5. $\beta \propto n^{-\iota_{1}}$ for some constant $\iota_{1} \in(0,1)$.

Assumptions A3 and A5 are standard in nonparametric density estimation. To make the statements of other assumptions clearer, we will give, in the next section, some examples which are covered (or not covered) in our framework of Assumption A1.1. Define

$$
\gamma_{1}(x ; f)=\zeta_{1,1} f^{(1)}(x)+\frac{\zeta_{1,2}}{2} x f^{(2)}(x), \quad \gamma_{2}(x ; f)=\frac{\zeta_{2,2}}{2} f^{(2)}(x)+\frac{\zeta_{2,3}}{6} x f^{(3)}(x)+\frac{\zeta_{2,4}}{24} x^{2} f^{(4)}(x) .
$$

Before presenting our main results on the JLN-type (Subsection 2.2), let us now illustrate the key quantities in our analysis (compared to (3) and (5)). For simplicity, we write

$$
B_{\beta}^{(K), f}(s)=\int_{\mathcal{S}} K(t ; s, \beta) f(t) d t-f(s), \quad s \in \mathcal{S} .
$$

As usual, Taylor's expansion around $t=s$ yields

$$
\left|\int_{\mathcal{S}} K(t ; s, \beta) f(t) d t-f(s)-\sum_{j=1}^{4} \frac{f^{(j)}(s)}{j !} \widetilde{\mu}_{j}(K(\cdot ; s, \beta))\right| \leq \frac{L_{4}}{24} \widetilde{\mu}_{6}^{\left(4+\eta_{4}\right) / 6}(K(\cdot ; s, \beta)),
$$


hence, for all sufficiently small $\beta>0, s \geq c_{L} \beta^{\eta}\left(\eta \in[0,1)\right.$ and $c_{L}>0$ are constants) implies that

$$
\begin{aligned}
\left|B_{\beta}^{(K), f}(s)-\sum_{j=1}^{2} \beta^{j} \gamma_{j}(s ; f)\right| & \leq \sum_{j=2}^{4} \frac{\| f^{(j)}|| \mathcal{S}_{S}}{j !}\left|r_{j, \beta}(s)\right|+\frac{L_{4}}{24}\left|r_{6, \beta}(s)\right|^{\left(4+\eta_{4}\right) / 6} \\
& \leq M^{\prime}\left[\beta^{3-\eta}+\beta^{2+\eta_{4} / 2}\left(1+s^{2+\eta_{4} / 2}\right)\right]
\end{aligned}
$$

for some constant $M^{\prime}>0$, independent of $\beta$ and $s$ (see Assumptions A1.3 and A2(i)). Our framework of Assumption A1.1-2 facilitates the boundary analysis when $s=\beta \kappa$ ( $\kappa \geq 0$ is a constant), i.e.,

$$
B_{\beta}^{(K), f}(\beta \kappa)=\beta\left\{\mu_{1}^{\prime}(\kappa)-\kappa\right\} f^{(1)}(0)+\frac{\beta^{2}}{2}\left\{\mu_{2}^{\prime}(\kappa)-\kappa^{2}\right\} f^{(2)}(0)+O\left(\beta^{3}\right)
$$

(for the expression (8), we can use $\mu_{1}^{\prime}(\kappa)-\kappa=\mu_{1}^{\prime}(0)$ under Assumption A1.3), since

$$
\left|\int_{\mathcal{S}} K(t ; \beta \kappa, \beta) f(t) d t-f(\beta \kappa)-\sum_{j=1}^{2} \frac{f^{(j)}(\beta \kappa)}{j !} \widetilde{\mu}_{j}(K(\cdot ; \beta \kappa, \beta))\right| \leq \frac{\left\|f^{(3)}\right\|_{\mathcal{S}}}{6} \widetilde{\mu}_{4}^{3 / 4}(K(\cdot ; \beta \kappa, \beta)),
$$

with $\widetilde{\mu}_{j}(K(\cdot ; \beta \kappa, \beta))=\beta^{j} \int_{\mathcal{S}}(u-\kappa)^{j} p_{K}(u ; \kappa) d u, j=1,2,4$ (note that $\left|f^{(2)}(\beta \kappa)-f^{(2)}(0)\right| \leq \beta \kappa\left\|f^{(3)}\right\|_{\mathcal{S}}$ and $\left.\left|f^{(1)}(\beta \kappa)-f^{(1)}(0)-\beta \kappa f^{(2)}(0)\right| \leq(1 / 2)(\beta \kappa)^{2}|| f^{(3)} \|_{\mathcal{S}}\right)$.

Remark 1 (i) $\sup _{x \in \mathcal{S}}\left|B_{\beta}^{(K), f}(x)\right| \leq 2\|f\|_{\mathcal{S}}$ under the boundedness of $f$ and Assumption A1.1.

(ii) Under Assumptions A1, A2(i), and A3(i), we have, for all sufficiently small $\beta>0$,

$$
\sup _{x \in[0, \widetilde{R}]}\left|B_{\beta}^{(K), f}(x)\right|=O(\beta) \quad \text { for any constant } \widetilde{R}>0 .
$$

Also,

$$
\sup _{x \in\left[0, \beta^{\tau}\right]}\left|B_{\beta}^{(K), f}(x)-\beta \xi_{1,1} f^{(1)}(0)\right|=O\left(\beta^{2 \tau}\right) \quad \text { for any constant } \tau \in(0,1) .
$$

Proof of Remark 1(ii) We see that, for $x \geq 0$,

$$
\left|\int_{\mathcal{S}} K(t ; x, \beta) f(t) d t-f(x)-\beta \xi_{1,1} f^{(1)}(x)\right| \leq \frac{\left\|f^{(2)}\right\|_{\mathcal{S}}}{2} \widetilde{\mu}_{2}(K(\cdot ; x, \beta)),
$$

hence, $\left|B_{\beta}^{(K), f}(x)\right| \leq \beta \xi_{1,1}|| f^{(1)}\left\|_{\mathcal{S}}+(1 / 2)\right\| f^{(2)} \|_{\mathcal{S}} \widetilde{\mu}_{2}(K(\cdot ; x, \beta))$. For any $\widetilde{R}>0$, Assumption A2(i) yields, for all sufficiently small $\beta>0$, $\sup _{x \in\left[\beta^{1 / 2}, \widetilde{R}\right]} \widetilde{\mu}_{2}(K(\cdot ; x, \beta)) \leq \beta \zeta_{1,2} \widetilde{R}+\beta^{2}\left(\left|\zeta_{2,2}\right|+M_{2}\right)$, whereas Assumption A1.2 implies that

$$
\widetilde{\mu}_{2}(K(\cdot ; x, \beta)) \leq 2\left[\int_{\mathcal{S}} t^{2} \frac{1}{\beta} p_{K}\left(\frac{t}{\beta} ; \frac{x}{\beta}\right) d t+x^{2}\right] \leq 2\left[\beta^{2}\left\{1+\left(\frac{x}{\beta}\right)^{2}\right\} \sup _{y \in \mathcal{S}} \frac{\mu_{2}^{\prime}(y)}{1+y^{2}}+x^{2}\right],
$$

hence, $\sup _{x \in\left[0, \beta^{\widetilde{\tau}}\right]} \widetilde{\mu}_{2}(K(\cdot ; x, \beta))=O\left(\beta^{\min (2,2 \widetilde{\tau})}\right)$ for any constant $\widetilde{\tau}>0$. The latter result then follows from $\sup _{x \in\left[0, \beta^{\widetilde{\tau}}\right]}\left|f^{(1)}(x)-f^{(1)}(0)\right| \leq \beta^{\widetilde{\tau}}|| f^{(2)} \|_{\mathcal{S}}$.

Remark 2 The error bound (7) should read

$$
\left|B_{\beta}^{(K), f}(s)-\sum_{j=1}^{2} \beta^{j} \gamma_{j}(s ; f)\right| \leq M^{\prime} \beta^{2+\eta_{4} / 2}\left(1+s^{2+\eta_{4} / 2}\right) \text { for } s \geq 0,
$$


if Assumption A2(i) can be replaced by the following stronger version ${ }^{[4]}$ :

A2. (i $\sharp)$ The $j$ th moments around $x \geq 0 ; \widetilde{\mu}_{j}(K(\cdot ; x, \beta)), j=2,3,4,6$, are given by

$$
\widetilde{\mu}_{j}(K(\cdot ; x, \beta))=\left\{\begin{array}{rl}
\beta \zeta_{1,2} x+\beta^{2} \zeta_{2,2} & j=2, \\
\beta^{2} \zeta_{2,3} x+r_{3, \beta}(x), j & =3, \\
\beta^{2} \zeta_{2,4} x^{2}+r_{4, \beta}(x), j & =4, \\
r_{6, \beta}(x), j & =6
\end{array}\right.
$$

with $\left|r_{3, \beta}(x)\right| \leq M_{3} \beta^{3},\left|r_{4, \beta}(x)\right| \leq M_{4} \beta^{3}(x+\beta)$, and $\left|r_{6, \beta}(x)\right| \leq M_{6} \beta^{3}(x+\beta)^{3}$ for some constants $\zeta_{1,2}, \zeta_{2,2}, \zeta_{2,3}, \zeta_{2,4}\left(\zeta_{1,2}, \zeta_{2,2}, \zeta_{2,4}>0\right)$ and $M_{3}, M_{4}, M_{6}>0$, independent of $\beta$ and $x$.

Also, if $g$ is continuously differentiable $g$ on $\mathcal{S}$, with $\sum_{i=0}^{1}\left\|g^{(i)}\right\|_{\mathcal{S}}<\infty$, Assumptions A1.1-2, A2(i,ii.2,iii), and A3(i) yield, for all sufficiently small $\beta>0$,

$$
\int_{\mathcal{S}} K^{2}(t ; x, \beta) g(t) d t=\left\{\begin{array}{l}
\beta^{-1 / 2} \frac{g(x)}{2 \sqrt{\pi x}}+o\left((\beta x)^{-1 / 2}\right)+O(1) \quad \text { for } x \in\left[c_{L} \beta^{\eta}, c_{U}\right], \\
\beta^{-1} g(0) \int_{\mathcal{S}} p_{K}^{2}(u ; \kappa) d u+O(1) \text { for } x=\beta \kappa,
\end{array}\right.
$$

where $\eta \in[0,1), 0<c_{L}<c_{U}$, and $\kappa \geq 0$ are constants. The proof is easy, as follows: For $x \in\left[c_{L} \beta^{\eta}, c_{U}\right]$, Taylor's expansion around $t=x$ yields

$$
\begin{aligned}
\left|\int_{\mathcal{S}} K^{2}(t ; x, \beta) g(t) d t-g(x) \int_{\mathcal{S}} K^{2}(t ; x, \beta) d t\right| & \leq\left\|g^{(1)}\right\|_{\mathcal{S}} \int_{\mathcal{S}}|t-x| K^{2}(t ; x, \beta) d t \\
& \leq\left\|g^{(1)}\right\|_{\mathcal{S}} \frac{C_{K}^{\prime}}{\sqrt{\beta x}} \widetilde{\mu}_{2}^{1 / 2}(K(\cdot ; x, \beta)) \\
& \leq\left\|g^{(1)}\right\|_{\mathcal{S}} C_{K}^{\prime}\left\{\zeta_{1,2}+\frac{\beta^{1-\eta}}{c_{L}}\left(\left|\zeta_{2,2}\right|+M_{2}\right)\right\}^{1 / 2},
\end{aligned}
$$

whereas, for $x=\beta \kappa$, Taylor's expansion around $t=0$ yields

$$
\left|\int_{\mathcal{S}} K^{2}(t ; \beta \kappa, \beta) g(t) d t-g(0) \int_{\mathcal{S}} K^{2}(t ; \beta \kappa, \beta) d t\right| \leq\left\|g^{(1)}\right\|_{\mathcal{S}} \int_{\mathcal{S}} t K^{2}(t ; \beta \kappa, \beta) d t,
$$

with $\int_{\mathcal{S}} t^{j} K^{2}(t ; \beta \kappa, \beta) d t=\beta^{j-1} \int_{\mathcal{S}} u^{j} p_{K}^{2}(u ; \kappa) d u, j=0,1$.

\subsection{Main results}

\subsubsection{Bias and variance approximations}

We are ready to present the bias and variance of the JLN-type bias-corrected KDE (6).

Proposition 1 Suppose that Assumptions A1, A2(i,ii.1), and A3-A5 hold (we set $\bar{\ell} \geq 3$ for A4(i) $)^{[5]}$, and that $\epsilon \propto \beta^{\iota_{2}}$ for some constant $\iota_{2}>1$. Then, for all sufficiently small $\beta>0$,

$$
\operatorname{Bias}\left[\widetilde{f}_{\beta, J L N}(x)\right]=\left\{\begin{array}{l}
\beta^{2} B_{J L N}(x ; f)+o\left(\beta^{2}\right)+O\left(n^{-1}(\beta x)^{-1 / 2}\right) \quad \text { for } x \in\left[c_{L} \beta^{\eta}, c_{U}\right], \\
\beta^{2} I(\kappa ; f)+o\left(\beta^{2}\right)+O\left(n^{-1} \beta^{-1}\right) \quad \text { for } x=\beta \kappa,
\end{array}\right.
$$

\footnotetext{
${ }^{[4]}$ The requirement of $\widetilde{\mu}_{2}(K(\cdot ; x, \beta))=\beta \zeta_{1,2} x+\beta^{2} \zeta_{2,2}$ is satisfied, if, in addition to Assumption A1.3, the 2nd raw moment $\mu_{2}^{\prime}(y)$, for $y \geq 0$, is a quadratic polynomial in $y ; \mu_{2}^{\prime}(y)=y^{2}+\left(2 \zeta_{1,1}+\zeta_{1,2}\right) y+\zeta_{2,2}$; in this case, we have $\zeta_{j, j}=\mu_{j}^{\prime}(0)>0, j=1,2$.

${ }^{[5]}$ Hirukawa and Sakudo (2014) (see also the subsequent papers; Funke and Kawka (2015), Hirukawa and Sakudo (2015), Zougab and Adjabi (2016), and Zougab et al. (2018)) assumed $n \beta^{3} \rightarrow \infty$ to control the remainder term of the bias, i.e., $O\left(n^{-1} \beta^{-1}\right)=o\left(b^{2}\right)$. Their stronger assumption is, however, redundant for analyzing the M(I)SE.
} 
where $\eta \in[0,1), 0<c_{L}<c_{U}$, and $\kappa \geq 0$ are constants,

$$
\begin{aligned}
B_{J L N}(x ; f) & =-f(x) \gamma_{1}\left(x ; \frac{\gamma_{1}(\cdot ; f)}{f(\cdot)}\right) \\
I(\kappa ; f) & =\left\{\mu_{1}^{\prime}(0)\right\}^{2} \frac{\left\{f^{(1)}(0)\right\}^{2}}{f(0)}+\left\{-\frac{1}{2} \int_{\mathcal{S}} \mu_{2}^{\prime}(y) p_{K}(y ; \kappa) d y+\mu_{2}^{\prime}(\kappa)-\frac{\kappa^{2}}{2}\right\} f^{(2)}(0) .
\end{aligned}
$$

Note that, under Assumption $\mathrm{A} 2\left(\mathrm{i}^{\sharp}\right)$, we can use

$$
I(\kappa ; f)=\zeta_{1,1}^{2} \frac{\left\{f^{(1)}(0)\right\}^{2}}{f(0)}-\zeta_{1,1}\left(\zeta_{1,1}+\frac{\zeta_{1,2}}{2}\right) f^{(2)}(0)=B_{J L N}(\beta \kappa ; f)+O(\beta) .
$$

Proposition 2 Suppose that Assumptions A1-A5 hold (we set $\bar{\ell} \geq 5$ for A4(i)), and that $\epsilon \propto \beta^{\iota_{2}}$ for some constant $\iota_{2} \geq 1$. Then, for all sufficiently small $\beta>0$,

$$
V\left[\widetilde{f}_{\beta, J L N}(x)\right]=\left\{\begin{array}{l}
n^{-1} \beta^{-1 / 2} \lambda(1 / 2) \frac{f(x)}{2 \sqrt{\pi x}}+o\left(n^{-1}(\beta x)^{-1 / 2}\right)+O\left(\beta^{5}\right) \quad \text { for } x \in\left[c_{L} \beta^{\eta}, c_{U}\right], \\
n^{-1} \beta^{-1} J(\kappa) f(0)+o\left(n^{-1} \beta^{-1}\right)+O\left(\beta^{5}\right) \quad \text { for } x=\beta \kappa,
\end{array}\right.
$$

where $\eta \in[0,1 / 4), 0<c_{L}<c_{U}$, and $\kappa \geq 0$ are constants, and $J(\kappa)=4 J_{1}(\kappa)-4 J_{2}(\kappa)+J_{3}(\kappa)$ with

$$
\begin{aligned}
& J_{1}(\kappa)=\int_{\mathcal{S}} p_{K}^{2}(u ; \kappa) d u, \quad J_{2}(\kappa)=\int_{\mathcal{S}} p_{K}(y ; \kappa) \int_{\mathcal{S}} p_{K}(u ; \kappa) p_{K}(u ; y) d u d y, \\
& J_{3}(\kappa)=\int_{\mathcal{S}} \int_{\mathcal{S}}\left[\prod_{j=1}^{2} p_{K}\left(y_{j} ; \kappa\right)\right] \int_{\mathcal{S}}\left[\prod_{j=1}^{2} p_{K}\left(u ; y_{j}\right)\right] d u d y_{1} d y_{2} .
\end{aligned}
$$

Note that $V\left[\widetilde{f}_{\beta, J L N}(x)\right]=O\left(n^{-1}(\beta x)^{-1 / 2}+\beta^{5}\right)$ for $x \in\left[c_{L} \beta^{\eta}, c_{U}\right]$, if $\eta \in[0,1)$.

Remark 3 A careful analysis of Proofs of Propositions 1 and 2 shows that, for all sufficiently small $\beta>0$,

$$
\begin{aligned}
\sup _{x \in\left[0, c_{U}\right]} V\left[\widetilde{f}_{\beta, J L N}(x)\right] & =O\left(n^{-1} \beta^{-1}+\beta^{5}\right), \\
\sup _{x \in\left[0, \beta^{\tau}\right]}\left|\operatorname{Bias}\left[\widetilde{f}_{\beta, J L N}(x)\right]\right| & =O\left(\beta^{2 \tau}+n^{-1} \beta^{-1}\right) \quad \text { for any constant } \tau \in(1 / 2,1) .
\end{aligned}
$$

These bounds will be required to show that the different rates in the variance and the remainder term of the bias has negligible impact on the truncated mean integrated squared error (MISE).

\subsubsection{MSE}

Propositions 1 and 2 (i.e., the bias (11) and variance (12)) immediately yield

$$
\begin{aligned}
& M S E\left[\tilde{f}_{\beta, J L N}\left(x_{0}\right)\right] \\
& = \begin{cases}\beta^{4} B_{J L N}^{2}\left(x_{0} ; f\right)+n^{-1} \beta^{-1 / 2} \lambda(1 / 2) \frac{f\left(x_{0}\right)}{2 \sqrt{\pi x_{0}}}+o\left(\beta^{4}+n^{-1} \beta^{-1 / 2}\right) & \text { for fixed } x_{0} \in\left(0, c_{U}\right], \\
\beta^{4} I^{2}(0 ; f)+n^{-1} \beta^{-1} J(0) f(0)+o\left(\beta^{4}+n^{-1} \beta^{-1}\right) & \text { for } x_{0}=0,\end{cases}
\end{aligned}
$$


whose leading term is minimized by choosing

$$
\beta_{J L N}^{o p t}\left(x_{0}\right)=\left\{\begin{array}{l}
{\left[\frac{\lambda(1 / 2) \frac{f\left(x_{0}\right)}{2 \sqrt{\pi x_{0}}}}{8 B_{J L N}^{2}\left(x_{0} ; f\right)} n^{-1}\right]^{2 / 9} \text { for fixed } x_{0} \in\left(0, c_{U}\right]} \\
{\left[\frac{J(0) f(0)}{4 I^{2}(0 ; f)} n^{-1}\right]^{1 / 5} \text { for } x_{0}=0}
\end{array}\right.
$$

and the optimal AMSE is then given by

$$
A M S E^{o p t}\left[\widetilde{f}_{\beta, J L N}\left(x_{0}\right)\right]=\left\{\begin{array}{l}
9\left\{B_{J L N}^{2}\left(x_{0} ; f\right)\right\}^{1 / 9}\left[\frac{1}{8} \lambda(1 / 2) \frac{f\left(x_{0}\right)}{2 \sqrt{\pi x_{0}}} n^{-1}\right]^{8 / 9} \text { for fixed } x_{0} \in\left(0, c_{U}\right], \\
5\left\{I^{2}(0 ; f)\right\}^{1 / 5}\left[\frac{1}{4} J(0) f(0) n^{-1}\right]^{4 / 5} \text { for } x_{0}=0 .
\end{array}\right.
$$

This order is faster, compared with (4) when $q=r=1$ and $r^{\prime}=1 / 2$.

\subsubsection{MISE}

To measure a global performance of the density estimator, we now technically use the truncated MISE, defined by $M I S E_{w}[\widehat{f}]=\int_{[0, w]} M S E[\widehat{f}(x)] d x$, where $w \in\left(0, c_{U}\right]$ is a constant. Propositions 1 and 2, together with Remark 3, yield $M I S E_{w}\left[\tilde{f}_{\beta, J L N}\right]=A M I S E_{w}\left[\tilde{f}_{\beta, J L N}\right]+o\left(\beta^{4}+n^{-1} \beta^{-1 / 2}\right)$, where

$$
A M I S E_{w}\left[\widetilde{f}_{\beta, J L N}\right]=\beta^{4} \int_{0}^{w} B_{J L N}^{2}(x ; f) d x+n^{-1} \beta^{-1 / 2} \lambda(1 / 2) \int_{0}^{w} \frac{f(x)}{2 \sqrt{\pi x}} d x
$$

(with $\beta \propto n^{-2 / 9}$, the convergence rate $n^{-8 / 9}$ is achieved, which is faster than that of the uncorrected case, $\left.n^{-4 / 5}\right)$.

Proof Choosing constants $\tau_{1} \in(4 / 5,1)$ and $\tau_{2} \in(0,1 / 4)$, we have, for all sufficiently small $\beta>0$,

$$
\begin{aligned}
\int_{\beta^{\tau_{1}}}^{w} \operatorname{Bias}^{2}\left[\tilde{f}_{\beta, J L N}(x)\right] d x & =\beta^{4} \int_{\beta^{\tau_{1}}}^{w} B_{J L N}^{2}(x ; f) d x+o\left(n^{-1} \beta^{-1 / 2}+\beta^{4}\right)+O\left(\left(n^{-1} \beta^{-1 / 2}\right)^{2} \log (1 / \beta)\right) \\
& =\beta^{4} \int_{0}^{w} B_{J L N}^{2}(x ; f) d x+o\left(n^{-1} \beta^{-1 / 2}+\beta^{4}\right), \\
\int_{\beta^{\tau_{2}}}^{w} V\left[\widetilde{f}_{\beta, J L N}(x)\right] d x & =n^{-1} \beta^{-1 / 2} \lambda(1 / 2) \int_{\beta^{\tau_{2}}}^{w} \frac{f(x)}{2 \sqrt{\pi x}} d x+o\left(n^{-1} \beta^{-1 / 2}+\beta^{4}\right) \\
& =n^{-1} \beta^{-1 / 2} \lambda(1 / 2) \int_{0}^{w} \frac{f(x)}{2 \sqrt{\pi x}} d x+o\left(n^{-1} \beta^{-1 / 2}+\beta^{4}\right), \\
\int_{0}^{\beta^{\tau_{1}}} M S E\left[\widetilde{f}_{\beta, J L N}(x)\right] d x & =O\left(\left\{\beta^{4 \tau_{1}}+\left(n^{-1} b^{-1}\right)^{2}+n^{-1} \beta^{-1}+\beta^{5}\right\} \beta^{\tau_{1}}\right)=o\left(n^{-1} \beta^{-1 / 2}+\beta^{4}\right), \\
\int_{\beta^{\tau_{1}}}^{\beta^{\tau_{2}}} V\left[\widetilde{f}_{\beta, J L N}(x)\right] d x & =O\left(n^{-1} \beta^{-1 / 2+\tau_{1} / 2}+\beta^{5+\tau_{2}}\right)=o\left(n^{-1} \beta^{-1 / 2}+\beta^{4}\right) .
\end{aligned}
$$

\section{Special cases and discussion}

To build the asymmetric kernel $K(\cdot ; x, \beta)$ supported on $[0, \infty)$, we focus on the application of three different families: 
- The modified Bessel (MB) density, with the parameter $\omega, \sigma>0$ and $\lambda \in \mathbb{R}$, is defined by

$$
K_{\omega, \sigma}^{(M B)}(s ; \lambda)=\frac{s^{\lambda-1}}{2 \sigma^{\lambda} K_{\lambda}(\omega)} \exp \left\{-\frac{\omega}{2}\left(\frac{s}{\sigma}+\frac{\sigma}{s}\right)\right\}
$$

where $K_{\nu}$ is the modified Bessel function of the third kind with index $\nu \in \mathbb{R}$, i.e.,

$$
K_{\nu}(\omega)=\int_{0}^{\infty} \frac{s^{\nu-1}}{2} \exp \left\{-\frac{\omega}{2}\left(s+s^{-1}\right)\right\} d s=\int_{0}^{\infty} \cosh (\nu t) \exp \{-\omega \cosh (t)\} d t
$$

(note that $K_{\nu}(\omega)=K_{-\nu}(\omega)$ and $\left.K_{1 / 2}(\omega)=\{\pi /(2 \omega)\}^{1 / 2} e^{-\omega}\right)$.

- The weighted $\operatorname{LN}[\lambda]$ density, with the parameter $\mu, \lambda \in \mathbb{R}$ and $\sigma^{2}>0$, is defined by

$$
K_{\mu, \sigma^{2}}^{(L N)}(s ; \lambda)=\frac{s^{\lambda-1}}{\sqrt{2 \pi} \sigma} \exp \left\{-\frac{(\log s-\mu)^{2}}{2 \sigma^{2}}-\lambda \mu-\frac{\lambda^{2} \sigma^{2}}{2}\right\}
$$

(by definition, $K_{\mu, \sigma^{2}}^{(L N)}(\cdot ; \lambda)=K_{\mu+\lambda \sigma^{2}, \sigma^{2}}^{(L N)}(\cdot ; 0)$ for $\left.\lambda \in \mathbb{R}\right)$.

- The Amoroso density, with the parameter $\alpha, \beta>0$ and $\gamma \neq 0$, is defined by

$$
K_{\alpha, \beta, \gamma}^{(A)}(s)=\frac{|\gamma| s^{\alpha \gamma-1}}{\beta^{\alpha \gamma} \Gamma(\alpha)} \exp \left\{-\left(\frac{s}{\beta}\right)^{\gamma}\right\}
$$

\subsection{Application to MIG/MLN/Amoroso KDEs}

Example 1 (MIG KDE) Reformulating the IG/BS/RIG KDEs due to Jin and Kawczak (2003) and Scaillet (2004), Igarashi and Kakizawa (2014b, 2015) suggested the mixture of IG and RIG kernels (in short, the MIG kernel)

$$
K^{\left(M I G_{p}\right)}(\cdot ; x, b)=(1-p) K_{x / b+1, x+b}^{(M B)}(\cdot ;-1 / 2)+p K_{x / b+1, x+b}^{(M B)}(\cdot ; 1 / 2), \quad x \geq 0
$$

to construct the (uncorrected) MIG KDE defined by $\widehat{f}_{b}^{\left(M I G_{p}\right)}(x)=n^{-1} \sum_{i=1}^{n} K^{\left(M I G_{p}\right)}\left(X_{i} ; x, b\right)$, where $p \in[0,1]$ is a mixing proportion, independent of $\beta$ and $x$. This class of estimators contains the (reformulated) IG/BS/RIG KDEs as special cases $p=0,1 / 2,1$, respectively.

Example 2 (MLN KDE) Reformulating the LN KDE due to Jin and Kawczak (2003), Igarashi (2016b) (see also Igarashi and Kakizawa (2015)) used the $\mathrm{LN}[ \pm 1 / 2] \operatorname{kernel} K_{\mu_{b}(x), \sigma_{b}^{2}(x)}^{(L N)}(\cdot ; \pm 1 / 2), x \geq 0$, where

$$
\mu_{b}(x)=\log (x+b)=\log b \rho(x / b), \quad \sigma_{b}^{2}(x)=\log \left(1+\frac{b}{x+b}\right)=\log \left\{1+\frac{1}{\rho(x / b)}\right\} \leq \log 2
$$

(we write $\rho(t)=t+1$ ). Parallel to the MIG kernel, we may choose a mixture of the $\mathrm{LN}[ \pm 1 / 2]$ kernels (in short, the MLN kernel)

$$
K^{\left(M L N_{p}\right)}(\cdot ; x, b)=(1-p) K_{\mu_{b}(x), \sigma_{b}^{2}(x)}^{(L N)}(\cdot ;-1 / 2)+p K_{\mu_{b}(x), \sigma_{b}^{2}(x)}^{(L N)}(\cdot ; 1 / 2), \quad x \geq 0
$$

to construct the (uncorrected) MLN KDE defined by $\widehat{f}_{b}^{\left(M L N_{p}\right)}(x)=n^{-1} \sum_{i=1}^{n} K^{\left(M L N_{p}\right)}\left(X_{i} ; x, b\right)$. 
Example 3 (Amoroso (or generalized gamma) KDE) For every constant $\gamma>0$, Igarashi and Kakizawa (2018a) suggested the Amoroso kernel

$$
K^{\left(A_{\gamma}\right)}(s ; x, b)=K_{\alpha_{b}(x), b \beta_{b}(x), \gamma}^{(A)}(s), \quad x \geq 0
$$

where

$$
\alpha_{b}(x)=\frac{x / b+1}{\gamma}=\frac{\rho(x / b)}{\gamma}, \quad \beta_{b}(x)=(x / b+1) \frac{\Gamma\left(\frac{x / b+1}{\gamma}\right)}{\Gamma\left(\frac{x / b+2}{\gamma}\right)}=\rho(x / b) \frac{\Gamma\left(\frac{\rho(x / b)}{\gamma}\right)}{\Gamma\left(\frac{\rho(x / b)+1}{\gamma}\right)} .
$$

In this paper, we use the (uncorrected) Amoroso KDE defined by $\widehat{f}_{b}^{\left(A_{\gamma}\right)}(x)=n^{-1} \sum_{i=1}^{n} K^{\left(A_{\gamma}\right)}\left(X_{i} ; x, b \gamma\right)$, for every constant $\gamma>0^{[6]}$. The gamma KDE due to Chen (2000), $\widehat{f}_{b}^{(G)}(x)=n^{-1} \sum_{i=1}^{n} K_{x / b+1, b, 1}^{(A)}(s)$ is a core member with $\gamma=1$.

Now, to ensure Propositions 1 and 2 for the bias-corrected MIG/MLN/Amoroso KDEs, defined by

$$
\widehat{f}_{b, J L N}^{(\#)}(x)=\left\{\widehat{f}_{b}^{(\#)}(x)+\epsilon\right\} \frac{1}{n} \sum_{i=1}^{n} \frac{K^{(\#)}\left(X_{i} ; x, b\right)}{\widehat{f}_{b}^{(\#)}\left(X_{i}\right)+\epsilon}, \quad x \geq 0, \quad \#=M I G_{p}, M L N_{p}, A_{\gamma},
$$

we have only to verify Assumptions on the respective kernel $K^{(\#)}(\cdot ; \cdot, b)$. The most of them is not restrictive or available from the existing literature. By construction, Assumptions A1 and A2(i,iii) can be readily verified for three examples. In addition to $q=r=1$ and $r^{\prime}=1 / 2$, from Lemma 1 of Igarashi and Kakizawa (2015) and Lemma A.1 of Igarashi and Kakizawa (2018a), we obtain

- $\left(\zeta_{1,1}^{\left(\#_{p}\right)}, \zeta_{1,2}^{\left(\#_{p}\right)}\right)=(p+1,1),\left(\zeta_{2,2}^{\left(\#_{p}\right)}, \zeta_{2,3}^{\left(\#_{p}\right)}, \zeta_{2,4}^{\left(\#_{p}\right)}\right)=(5 p+2,3(p+2), 3)$, where $\#=M I G, M L N$,

- $\left(\zeta_{1,1}^{\left(A_{\gamma}\right)}, \zeta_{1,2}^{\left(A_{\gamma}\right)}\right)=(\gamma, 1),\left(\zeta_{2,2}^{\left(A_{\gamma}\right)}, \zeta_{2,3}^{\left(A_{\gamma}\right)}, \zeta_{2,4}^{\left(A_{\gamma}\right)}\right)=\left(\frac{1}{2}\left(3 \gamma^{2}+1\right), 2 \gamma+3,3\right)$.

Assumption A2 $\left(\mathrm{i}^{\sharp}\right)$ holds for the MIG/LN[-1/2]/gamma kernels ${ }^{[7]}$. The uniform/nonuniform bounds in Assumption A2(ii) for the MB kernel $K_{x / b+1, x+b}^{(M B)}(\cdot ; \lambda)$ and the weighted LN kernel $K_{\mu_{b}(x), \sigma_{b}^{2}(x)}^{(L N)}(\cdot ; \lambda)$, where $\lambda \in \mathbb{R}$, are found in Lemma A.2 of Igarashi and Kakizawa (2014b), Lemma 4 of Igarashi (2016b), and Remark A.1(i) of Igarashi and Kakizawa (2015). See also Lemma A.3 of Igarashi and Kakizawa (2018a) for the Amoroso kernel $K^{\left(A_{\gamma}\right)}(\cdot ; \cdot, b \gamma)$.

On the other hand, after some algebra, it is straightforward to see that

\footnotetext{
${ }^{[6]}$ Igarashi and Kakizawa's original definition should read $n^{-1} \sum_{i=1}^{n} K^{\left(A_{\gamma}\right)}\left(X_{i} ; x, b\right)$.

In their paper (Igarashi and Kakizawa (2018a)), the negative exponent $\gamma<0$ has been allowed, for which the definition of the parameter $\left(\alpha_{b}(x), \beta_{b}(x)\right)$ should read, as follows: For every constant $\gamma<0$,

$$
\alpha_{b}(x)=\frac{\rho^{*}(x / b)+1}{|\gamma|}, \quad \beta_{b}(x)=\rho^{*}(x / b) \frac{\Gamma\left(\frac{\rho^{*}(x / b)+1}{|\gamma|}\right)}{\Gamma\left(\frac{\rho^{*}(x / b)}{|\gamma|}\right)}, \quad \text { where } \rho^{*}(x)=x+c^{*}, \text { with } c^{*}>1\left(\text { rather than } c^{*}=1\right) .
$$
}

For the simulation studies in Section $6, c^{*}=1.1$ was chosen.

${ }^{[7]}$ Let $x \geq 0$. We can see that $\widetilde{\mu}_{j}\left(K^{(\#)}(\cdot ; x, b)\right)$ 's, where \# $=G, M I G_{p}$, are the polynomials in $x$;

$$
\widetilde{\mu}_{j}\left(K^{(G)}(\cdot ; x, b)\right)=\left\{\begin{array}{ll}
b, & j=1, \\
b x+2 b^{2}, & j=2, \\
5 b^{2} x+O\left(b^{3}\right), & j=3, \\
3 b^{2} x^{2}+O\left(b^{3}(x+b)\right), & j=4, \\
O\left(b^{3}(x+b)^{3}\right), & j=6
\end{array} \text { and } \widetilde{\mu}_{j}\left(K^{\left(M I G_{p}\right)}(\cdot ; x, b)\right)= \begin{cases}(p+1) b, & j=1, \\
b x+(5 p+2) b^{2}, & j=2, \\
3(p+2) b^{2} x+O\left(b^{3}\right), & j=3, \\
3 b^{2} x^{2}+O\left(b^{3}(x+b)\right), & j=4, \\
O\left(b^{3}(x+b)^{3}\right), & j=6,\end{cases}\right.
$$


- the kernels $K^{\left(M I G_{p}\right)}(\cdot ; \cdot, b), K^{\left(M L N_{p}\right)}(\cdot ; \cdot, b)$, and $K^{\left(A_{\gamma}\right)}(\cdot ; \cdot, b \gamma)$ can be well approximated by a Gaussian, i.e., choosing constants $\eta \in[0,1 / 4)$ and $\tau \in((2 \eta+1) / 3,1 / 2)$, we have, for all sufficiently small $\beta>0$,

$$
\sqrt{\beta x} K\left(x+\sqrt{\beta x} z_{1} ; x+\sqrt{\beta x} z_{2}, \beta\right)=\phi\left(z_{1}-z_{2}\right)[1+o(1)]
$$

uniformly in $z_{1}, z_{2} \in\left[-\beta^{\tau} / \sqrt{\beta x}, \beta^{\tau} / \sqrt{\beta x}\right]$ and $x \in\left[c_{L} \beta^{\eta}, c_{U}\right]$.

This Gaussian approximation is a sufficient condition for Assumption A2(iv); note that, in this case, two coefficients $\zeta_{1,2}$ and $\zeta_{2,4}$ are equal to 1 and 3, respectively.

It remains to verify Assumption A4. For this, we now consider the two situations (we do not pursue more general conditions) where either of the following assumptions holds for some constant $\iota>0$ :

f1[८]. $\inf _{x \in \mathcal{S}}\left\{f(x) \exp \left(C_{2} x^{\iota}\right)\right\} \geq C_{1}$ for some constants $C_{1}, C_{2}>0$.

f2. $\inf _{x \in \mathcal{S}}\left\{f(x)(1+x)^{C_{2}}\right\} \geq C_{1}$ for some constants $C_{1}>0$ and $C_{2}>1$.

In either case, $\inf _{0 \leq x \leq R} f(x)>0$ for any $R>0$ (Assumption A4(i.1) is automatically satisfied).

Lemma 3 (i) If either of Assumption $\mathrm{f} 1[\iota]$ or $\mathrm{f} 2$ holds for some $\iota \in(0,1]$, Assumption A4(i.2,ii) holds for any $\bar{\ell} \in \mathbb{N}$ when $K$ is the MIG kernel $K^{\left(M I G_{p}\right)}(\cdot ; \cdot, b)$.

(ii) If Assumption $\mathrm{f} 2$ holds, Assumption A4(i.2,ii) holds for any $\bar{\ell} \in \mathbb{N}$ when $K$ is the MLN kernel $K^{\left(M L N_{p}\right)}(\cdot ; \cdot, b)$.

(iii) Given $\gamma>0$, if either of Assumption $\mathrm{f} 1[\iota]$ or $\mathrm{f} 2$ holds for some $\iota \in(0, \gamma]$, Assumption A4(i.2,ii) holds for any $\bar{\ell} \in \mathbb{N}$ when $K$ is the Amoroso kernel $K^{\left(A_{\gamma}\right)}(\cdot ; \cdot, b \gamma)$.

\subsection{Discussion}

Examples 1-3 have in common that the linear function $\rho(t)=t+1$ (one can use $\rho(t)=t+c$, where $c \geq 1$ is a constant) is adopted. As demonstrated in Chen (2000) and Igarashi and Kakizawa (2014b, 2015, 2018a) (see also Hirukawa and Sakudo (2015) for a subfamily of the generalized gamma KDE under an additional restriction $\gamma \geq 1$, focusing on the Nakagami case with $\gamma=2$ ), it may be true that

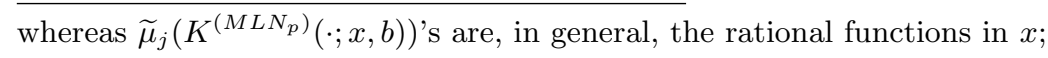

$$
\widetilde{\mu}_{j}\left(K^{\left(M L N_{p}\right)}(\cdot ; x, b)\right)= \begin{cases}(p+1) b, & j=1, \\ b x+(5 p+2) b^{2}+\frac{p b^{3}}{x+b}, & j=2, \\ 3(p+2) b^{2} x+r_{3, b}^{\left(M L N_{p}\right)}(x), & j=3, \\ 3 b^{2} x^{2}+r_{4, b}^{\left(M L N_{p}\right)}(x), & j=4, \\ r_{6, b}^{\left(M L N_{p}\right)}(x) & j=6\end{cases}
$$

with $\left|r_{3, b}^{\left(M L N_{p}\right)}(x)\right| \leq M_{3} b^{3},\left|r_{4, b}^{\left(M L N_{p}\right)}(x)\right| \leq M_{4} b^{3}(x+b)$, and $\left|r_{6, b}^{\left(M L N_{p}\right)}(x)\right| \leq M_{6} b^{3}(x+b)^{3}$ for some constants $M_{3}, M_{4}, M_{6}>0$, independent of $b$ and $x$.

The $\mathrm{MLN}_{p}$ kernel for $p \in(0,1]$ does not satisfy Assumption A2(i $\mathrm{i}^{\sharp}$. 
the use of two-regime $\rho$-function, having the form

$$
\rho_{c}(t)=\left\{\begin{array}{l}
c+t, t>2, \\
r_{c}(t), t \in[0,2]
\end{array} \quad\left(\text { we assume } c+2=r_{c}(2) \geq r_{c}(0) \geq 1 \text { for some constant } c \in \mathbb{R}\right),\right.
$$

where the function $\rho_{c}$ is continuous and non-decreasing on $[0, \infty)$, has the following advantages for the resulting estimator $\widetilde{f}_{b}^{\star}\left(\cdot ; \rho_{c}\right)$ (we call the two-regime type): (i) choosing $c=0, \operatorname{Bias}\left[\widetilde{f}_{b}^{\star}\left(x ; \rho_{0}\right)\right]$ does not involve $f^{\prime}$ in the leading $O(b)$-term, when $x \in[2 b, \infty)$, or (ii) one can minimize the $O\left(n^{-4 / 5}\right)$-MISE of $\widetilde{f}_{b}^{\star}\left(\cdot ; \rho_{c}\right)$ with respect to the additional parameter $c$; see Igarashi and Kakizawa (2014b). However, Assumption A1.3 is violated, due to the introduction of the non-linear $\rho$-function.

Remark 4 Even if Assumption A1.3 is replaced by the following assumption, (9) remains valid:

A1. $3^{\sharp} \cdot \mu_{1}^{\prime}(y)$ has the form ${ }^{[8]}$ of

$$
\mu_{1}^{\prime}(y)=\left\{\begin{array}{ll}
\zeta_{1,1}+y, & y>c^{\prime}, \\
\zeta(y), & y \in\left[0, c^{\prime}\right]
\end{array} \quad \text { for some constants } c^{\prime}>0 \text { and } \zeta_{1,1} \in \mathbb{R}\right.
$$

where $\zeta$ is a continuous and non-decreasing function on $[0, \infty)$, with $\zeta\left(c^{\prime}\right)=\zeta_{1,1}+c^{\prime} \geq \zeta(0)>0$.

The proof is easy: We see that, for $x \geq 0$,

$$
\left|\int_{\mathcal{S}} K(t ; x, \beta) f(t) d t-f(x)-\beta\left\{\mu_{1}^{\prime}(x / \beta)-x / \beta\right\} f^{(1)}(x)\right| \leq \frac{\left\|f^{(2)}\right\|_{\mathcal{S}}}{2} \widetilde{\mu}_{2}(K(\cdot ; x, \beta)),
$$

where $\sup _{x \in[0, \widetilde{R}]} \widetilde{\mu}_{2}(K(\cdot ; x, \beta))=O(\beta)$ for any constant $\widetilde{R}>0$ (see Proof of (9)). By definition, $x \in\left[c^{\prime} \beta, \widetilde{R}\right]$ implies $\mu_{1}^{\prime}(x / \beta)-x / \beta \equiv \zeta_{1,1}$. Also, $\sup _{x \in\left[0, c^{\prime} \beta\right]}\left|\mu_{1}^{\prime}(x / \beta)-x / \beta\right| \leq \zeta\left(c^{\prime}\right)+c^{\prime}$.

The following result reveals the weakness of the two-regime type.

Proposition 4 (Violation of Assumption A1.3) In Propositions 1 and 2, if Assumption A1.3 is replaced by $\mathrm{A} 1.3^{\sharp}$, then, in general, the order of the bias can not be improved near the boundary, i.e., the asymptotic bias (11) when $x=\beta \kappa$ should read as

$$
\operatorname{Bias}\left[\widetilde{f}_{\beta, J L N}(\beta \kappa)\right]=\beta\left[\left\{\mu_{1}^{\prime}(\kappa)-\kappa\right\}-\int_{\mathcal{S}}\left\{\mu_{1}^{\prime}(y)-y\right\} p_{K}(y ; \kappa) d y\right] f^{(1)}(0)+O\left(\beta^{2}+n^{-1} \beta^{-1}\right)
$$

(without a shoulder condition $f^{(1)}(0)=0$, Assumption A1.3 $3^{[9]}$ plays a crucial role of the bias reduction).

\footnotetext{
${ }^{[8]}$ Using the non-linear $\rho$-function $\rho_{c}$ (rather than $\left.\rho(t)=t+1\right)$, the two-regime $\mathrm{MIG}_{p} / \mathrm{MLN}_{p}$ kernels, for $p \in[0,1]$, yield $\mu_{1}^{\prime}(y)=p+\rho_{c}(y)$, whereas the two-regime Amoroso $\gamma$ kernel yields $\mu_{1}^{\prime}(y)=\gamma \rho_{c}(y / \gamma)$.

${ }^{[9]}$ Generally speaking, it is difficult to solve the integral equation $\mathcal{M}(\kappa)-\int_{\mathcal{S}} \mathcal{M}(y) p_{K}(y ; \kappa) d y=0$ with respect to the function $\mathcal{M}$; of course, $\mathcal{M}(\cdot) \equiv$ constant (hence, the case $\left.\mu_{1}^{\prime}(y)-y \equiv \zeta_{1,1}\right)$ is an exceptional solution.

Anyway, letting $r_{c}(t)=(c+1)(t / 2)^{2 /(c+1)}+1$, where $c>-1$, we numerically verify that $\mu_{1}^{\prime}(y)-y=p+\rho_{c}(y)-y$ for the two-regime $\mathrm{MIG}_{p} / \mathrm{MLN}_{p}$ kernels is not the solution of the integral equation; in this case, the JLN-type bias correction does not work, unless $f^{(1)}(0)=0$. The same argument is valid for the two-regime Amoroso $\gamma$ kernel by considering the case $\mu_{1}^{\prime}(y)=\gamma \rho_{c}(y / \gamma)$.
} 
We close this section by pointing out that, in the literature, the variants of the IG/BS/LN KDEs using $K^{(I G)_{S}}(s ; x, b)=K_{1 /(b x), x}^{(M B)}(s ;-1 / 2), K^{(B S)_{J K}}(s ; x, b)=(1 / 2)\left\{K_{1 / b, x}^{(M B)}(s ;-1 / 2)+K_{1 / b, x}^{(M B)}(s ; 1 / 2)\right\}$, and $K^{(L N)_{J K}}(s ; x, b)=K_{\log x, \log (1+b)}^{(L N)}(s ; 0)$, due to Jin and Kawczak (2003) ${ }^{[10]}$ and Scaillet (2004), have been discussed, but the resulting estimators yield $\widehat{f}_{b}(0)=0$ by construction. Clearly, such an unrealistic constraint is not suitable for estimating the density $f(0)>0$. See also Koul and Song (2013), Marchant et al. (2013), and Saulo et al. (2013). To make matters worse, a variant of the RIG KDE using $K^{(R I G)_{S}}(s ; x, b)=K_{(x-b) / b, x-b}^{(M B)}(s ; 1 / 2)$, due to Scaillet (2004), had the downward bias $\left(e^{-2}-1\right) f(0)$ at $x=0$; see Igarashi and Kakizawa (2014b). Also, the $\mathrm{IG}_{S} / \mathrm{BS}_{J K} / \mathrm{LN}_{J K} \mathrm{KDEs}$ had the asymptotic variance $n^{-1} b^{-1 / 2}\left(2 \sqrt{\pi} x^{J}\right)^{-1} f(x), J=1,3 / 2$ (rather than $n^{-1} b^{-1 / 2}(2 \sqrt{\pi x})^{-1} f(x)$ ) under $\int_{0}^{\infty} x^{-J} f(x) d x<\infty$ (any bounded continuous density $f$ on $[0, \infty$ ), with $f(0)>0$, was implicitly excluded). These problems were apparently caused by the bad parameterization; when $x=0$, the parameter $(1 /(b x), x),(1 / b, x),(\log x, \log (1+b))$ lies outside the parameter space of the IG/BS/LN density, respectively, and, when $x \in[0, b], K^{(R I G)_{S}}(\cdot ; x, b)$ is not the density. That is the reason why the authors have so far suggested a suitable parameterization for a certain parametric family $K_{\theta}(\cdot)$; see Examples 1-3, in such a way that, choosing a subcomponent $\theta_{1}$ to be a function $\theta_{1}(x, b)$ of the location $x \in \mathcal{S}$ and a smoothing parameter $b$, the resulting density estimator $n^{-1} \sum_{i=1}^{n} K_{\theta_{1}(x, b), \theta_{2}}\left(X_{i}\right)$ shares common properties to the gamma KDE (Chen (2000)). See also Kakizawa (2018).

Remark 5 The above-mentioned "bad" asymmetric KDEs may be applied, if $f(0)=0$ is known in advance; in this case, the corresponding "bad" kernels $\left(\mathrm{IG}_{S} / \mathrm{RIG}_{S} / \mathrm{BS}_{J K} / \mathrm{LN}_{J K}\right)$ share similar properties to $K(\cdot ; x, \beta)$ for fixed $x \in\left(0, c_{U}\right]$ (the details are omitted here). We stress that, after the JLN-type bias correction, the asymptotic variances at $x \in\left(0, c_{U}\right]$ increase with the factor $\lambda(1 / 2)$, hence, incorrect asymptotic variances of Theorem 2 in Hirukawa and Sakudo (2014) (see also Funke and Kawka (2015)) should be corrected as $n^{-1} b^{-1 / 2} \lambda(1 / 2)\left(2 \sqrt{\pi} x^{J}\right)^{-1} f(x)$.

\section{The case $[0, \infty]^{d}$ : product-type asymmetric KDE}

Once the univariate case is studied in detail, the product kernel method is available for estimating the multivariate density. To illustrate it, we focus on the situation where the data $\mathbf{X}_{i}=\left(X_{i 1}, \ldots, X_{i d}\right)^{\prime}$ is supported on $\mathcal{S}_{d}=[0, \infty)^{d}$, and construct $d$-variate product-type asymmetric KDE, defined by ${ }^{[11]}$

$$
\widetilde{f}_{\beta}^{\Pi}(\mathbf{x})=\frac{1}{n} \sum_{i=1}^{n} \prod_{j=1}^{d} K\left(X_{i j} ; x_{j}, \beta\right), \quad \mathbf{x}=\left(x_{1}, \ldots, x_{d}\right)^{\prime} \in \mathcal{S}_{d} .
$$

Similar theoretical results as in one dimension can be easily derived under the following assumptions:

- $\left(\mathrm{i}_{d}\right)$ A random sample $\left\{\mathbf{X}_{1}, \ldots, \mathbf{X}_{n}\right\}$ is drawn from an unknown density $f$ with support $\mathcal{S}_{d}$.

\footnotetext{
${ }^{[10]}$ Jin and Kawczak (2003) originally considered $K_{\log x, 4 \log (1+b)}^{(L N)}(s ; 0)$; but the "4" in their definition of $4 \log (1+b)$ seemed to be not important. Their estimator should read as $K_{\log x, \log (1+b)}^{(L N)}(s ; 0)$ (of course, $K_{\log x, b}^{(L N)}(s ; 0)$ can be used).

${ }^{[11]}$ Although, in this paper, we adopt the single smoothing parameter $\beta$ for simplicity, one can use vectors of the smoothing parameter proportional to a given vector $\left(\widetilde{c}_{1}, \ldots, \widetilde{c}_{d}\right)^{\prime}$, i.e., $\left(\beta_{1}, \ldots, \beta_{d}\right)^{\prime}=\beta\left(\widetilde{c}_{1}, \ldots, \widetilde{c}_{d}\right)^{\prime}$, where $\widetilde{c}_{j}>0$ is a constant.
} 
$\left(\mathrm{ii}_{d}\right)$ There exists a function $\varphi$, being four times continuously differentiable on $(\underline{c}, \infty)^{d}\left(\supset \mathcal{S}_{d}\right)$, such that the target density $f$ is the restriction of $\varphi$ on $\mathcal{S}_{d}$, and that $f$ and $\partial_{i_{1}} \cdots \partial_{i_{q}} f$ are all bounded for $q=1,2,3,4 ; i_{1}, \ldots, i_{q} \in\{1, \ldots, d\}$, where $\partial_{j}=\partial / \partial x_{j}$. In addition, there exist constants $\eta_{4} \in(0,1]$ and $L_{4}>0$, such that $\left|\partial_{i_{1}} \partial_{i_{2}} \partial_{i_{3}} \partial_{i_{4}} f(\mathbf{u})-\partial_{i_{1}} \partial_{i_{2}} \partial_{i_{3}} \partial_{i_{4}} f(\mathbf{v})\right| \leq L_{4}\|\mathbf{u}-\mathbf{v}\|^{\eta_{4}}$ for any $\mathbf{u}, \mathbf{v} \in \mathcal{S}_{d}$, where $\|\mathbf{u}\|$ is an Euculidian norm $\left(\sum_{j=1}^{d} u_{j}^{2}\right)^{1 / 2}$ of $\mathbf{u}=\left(u_{1}, \ldots, u_{d}\right)^{\prime}$.

(iii $\left.{ }_{d}\right) \beta \propto n^{-\iota_{1}}$ for some constant $\iota_{1} \in(0,1 / d)$.

Note that one technical assumption, analogous to Assumption A4 (see Lemma 3 in the previous section), can be verified under either of the following assumptions for some constant $\iota>0$ :

$\mathrm{f1}_{d}[\iota] . \inf _{\mathbf{x} \in \mathcal{S}_{d}}\left\{f(\mathbf{x}) \exp \left(C_{2}\|\mathbf{x}\|^{\iota}\right)\right\} \geq C_{1}$ for some constants $C_{1}, C_{2}>0$.

f2 $2_{d} . \inf _{\mathbf{x} \in \mathcal{S}_{d}}\left\{f(\mathbf{x})(1+\|\mathbf{x}\|)^{C_{2}}\right\} \geq C_{1}$ for some constants $C_{1}>0$ and $C_{2}>1$.

In either case, $\inf _{\mathrm{x} \in[0, R]^{d}} f(\mathbf{x})>0$ for any $R>0$. Other assumptions related to the product-type asymmetric kernel $K^{\Pi}(\mathbf{s} ; \mathbf{x}, \beta)=\prod_{j=1}^{d} K\left(s_{j} ; x_{j}, \beta\right)$ are met, provided that the selected asymmetric kernel $K(\cdot ; \cdot, \beta)$ in one dimensional case satisfies Assumptions A1 and A2. Here, the $j_{1}, \ldots, j_{d}$-th moment around $\mathbf{x}\left(\in \mathcal{S}_{d}\right)$ of $K^{\Pi}(\cdot ; \mathbf{x}, \beta)$, denoted by

$$
\widetilde{\mu}_{j_{1}, \ldots, j_{d}}\left(K^{\Pi}(\cdot ; \mathbf{x}, \beta)\right)=\int_{\mathcal{S}_{d}}\left(t_{1}-x_{1}\right)^{j_{1}} \cdots\left(t_{d}-x_{d}\right)^{j_{d}} K^{\Pi}(\mathbf{t} ; \mathbf{x}, \beta) d \mathbf{t} \quad \text { (if it exists), }
$$

can be written as

$$
\widetilde{\mu}_{j_{1}, \ldots, j_{d}}\left(K^{\Pi}(\cdot ; \mathbf{x}, \beta)\right)=\prod_{i=1}^{d} \widetilde{\mu}_{j_{i}}\left(K\left(\cdot ; x_{i}, \beta\right)\right) \quad \text { due to the independence, }
$$

so that the cross-moments up to the fourth-order, except for the marginal moments, are given by

$$
\begin{aligned}
\widetilde{\mu}_{j \text { times }}^{1, \ldots, 1,0, \ldots, 0}\left(K^{\Pi}(\cdot ; \mathbf{x}, \beta)\right) & =\left(\beta \zeta_{1,1}\right)^{j}, \quad j=2,3,4, \\
\widetilde{\mu}_{2,1,0, \ldots, 0}\left(K^{\Pi}(\cdot ; \mathbf{x}, \beta)\right) & =\beta^{2} \zeta_{1,1} \zeta_{1,2} x_{1} x_{2}+O\left(\beta^{3}\right) \quad \text { when } x_{1} / \beta \rightarrow \infty, \\
\widetilde{\mu}_{3,1,0, \ldots, 0}\left(K^{\Pi}(\cdot ; \mathbf{x}, \beta)\right) & =\widetilde{\mu}_{2,1,1,0, \ldots, 0}\left(K^{\Pi}(\cdot ; \mathbf{x}, \beta)\right)=O\left(\beta^{3} x_{1}\right) \quad \text { when } x_{1} / \beta \rightarrow \infty, \\
\widetilde{\mu}_{2,2,0, \ldots, 0}\left(K^{\Pi}(\cdot ; \mathbf{x}, \beta)\right) & =\beta^{2} \zeta_{1,2}^{2} x_{1} x_{2}+O\left(\beta^{3}\left(x_{1}+x_{2}\right)\right) \quad \text { when } x_{1} / \beta \rightarrow \infty \text { and } x_{2} / \beta \rightarrow \infty
\end{aligned}
$$

(there are, of course, the permutation variants, being omitted here). Similarly, the uniform/nonuniform bounds and the approximations of certain integrals (see Assumption A2(ii-iv)) are readily extended:

- $\sup _{\mathbf{x} \in \mathcal{S}_{d}} \sup _{\mathbf{s} \in \mathcal{S}_{d}} K^{\Pi}(\mathbf{s} ; \mathbf{x}, \beta) \leq\left(C_{K} \beta^{-1}\right)^{d}$,

- whenever $x_{1}, \ldots, x_{d^{\prime}}>0, \sup _{x_{d^{\prime}+1}, \ldots, x_{d} \in \mathcal{S}} \sup _{\mathbf{s} \in \mathcal{S}_{d}} K^{\Pi}(\mathbf{s} ; \mathbf{x}, \beta) \leq\left(C_{K} \beta^{-1}\right)^{d-d^{\prime}} \prod_{j=1}^{d^{\prime}}\left(C_{K}^{\prime} \beta x_{j}\right)^{-1 / 2}$, where $d^{\prime}=1, \ldots, d$ (the permutation variants for $\left(x_{1}, \ldots, x_{d}\right)$ are omitted here),

- given constants $\eta \in[0,1)$ and $c_{L}>0$, for all sufficiently small $\beta>0, x_{1}, \ldots, x_{d} \geq c_{L} \beta^{\eta}$ imply that

$$
\left|\int_{\mathcal{S}_{d}}\left\{K^{\Pi}(\mathbf{s} ; \mathbf{x}, \beta)\right\}^{2} d \mathbf{s}-\frac{\beta^{-d / 2}}{\prod_{j=1}^{d}\left(2 \sqrt{\pi x_{j}}\right)}\right| \leq \frac{\beta^{-d / 2} M_{d}}{\prod_{j=1}^{d} \sqrt{x_{j}}} \sum_{j=1}^{d} \frac{\beta}{x_{j}+\beta}
$$

for some constant $M_{d}>0$, independent of $\beta$ and $\mathbf{x}$, and 
- given constants $\eta \in[0,1 / 4)$ and $0<c_{L}<c_{U}$, for all sufficiently small $\beta>0$, the following approximations hold for $x_{1}, \ldots, x_{d} \in\left[c_{L} \beta^{\eta}, c_{U}\right]$ :

$$
\begin{aligned}
\int_{\mathcal{S}_{d}} K^{\Pi}(\mathbf{t} ; \mathbf{x}, \beta) \int_{\mathcal{S}_{d}} K^{\Pi}(\mathbf{s} ; \mathbf{x}, \beta) K^{\Pi}(\mathbf{s} ; \mathbf{t}, \beta) d \mathbf{s} d \mathbf{t} & =\frac{\beta^{-d / 2}}{\prod_{j=1}^{d}\left(\sqrt{6 \pi x_{j}}\right)}+o\left(\beta^{-d / 2} \prod_{j=1}^{d} x_{j}^{-1 / 2}\right), \\
\int_{\mathcal{S}_{d}} \int_{\mathcal{S}_{d}}\left\{\prod_{j=1}^{2} K^{\Pi}\left(\mathbf{t}_{j} ; \mathbf{x}, \beta\right)\right\} \int_{\mathcal{S}_{d}}\left\{\prod_{j=1}^{2} K^{\Pi}\left(\mathbf{s} ; \mathbf{t}_{j}, \beta\right)\right\} d \mathbf{s} d \mathbf{t}_{1} d \mathbf{t}_{2} & =\frac{\beta^{-d / 2}}{\prod_{j=1}^{d}\left(2 \sqrt{2 \pi x_{j}}\right)}+o\left(\beta^{-d / 2} \prod_{j=1}^{d} x_{j}^{-1 / 2}\right) .
\end{aligned}
$$

It turns out that the JLN-type bias-corrected estimator

$$
\widetilde{f}_{\beta, J L N}^{\Pi}(\mathbf{x})=\left\{\widetilde{f}_{\beta}^{\Pi}(\mathbf{x})+\epsilon\right\} \frac{1}{n} \sum_{i=1}^{n} \frac{K^{\Pi}\left(\mathbf{X}_{i} ; \mathbf{x}, \beta\right)}{\widetilde{f}_{\beta}^{\Pi}\left(\mathbf{X}_{i}\right)+\epsilon}, \quad \mathbf{x} \in \mathcal{S}_{d}
$$

(we assume $\epsilon \propto \beta^{\iota_{2}}$ for some $\iota_{2}>1$ ) has the asymptotic bias and variance at $\mathbf{x}=\left(x_{1}, \ldots, x_{d}\right)^{\prime}$, as follows: For all sufficiently small $\beta>0$, we have, for $x_{1}, \ldots, x_{d} \in\left[c_{L} \beta^{\eta}, c_{U}\right]$,

$$
\begin{gathered}
\operatorname{Bias}\left[\widetilde{f}_{\beta, J L N}^{\Pi}(\mathbf{x})\right]=-\beta^{2} f(\mathbf{x}) \gamma_{1, d}\left(\mathbf{x} ; \frac{\gamma_{1, d}(\cdot ; f)}{f(\cdot)}\right)+o\left(\beta^{2}\right)+O\left(n^{-1} \beta^{-d / 2} \prod_{j=1}^{d} x_{j}^{-1 / 2}\right), \quad \text { if } \eta \in[0,1), \\
V\left[\widetilde{f}_{\beta, J L N}^{\Pi}(\mathbf{x})\right]=n^{-1} \beta^{-d / 2} \frac{\lambda_{d} f(\mathbf{x})}{\prod_{j=1}^{d}\left(2 \sqrt{\pi x_{j}}\right)}+o\left(n^{-1} \beta^{-d / 2} \prod_{j=1}^{d} x_{j}^{-1 / 2}\right)+O\left(\beta^{5}\right), \quad \text { if } \eta \in[0,1 / 4)
\end{gathered}
$$

(note that, if $\eta \in[0,1)$, then, $V\left[\widetilde{f}_{\beta, J L N}^{\Pi}(\mathbf{x})\right]=O\left(n^{-1} \beta^{-d / 2} \prod_{j=1}^{d} x_{j}^{-1 / 2}+\beta^{5}\right)$ ), where

$$
\gamma_{1, d}(\mathbf{x} ; f)=\sum_{j=1}^{d}\left\{\zeta_{1,1} \partial_{j} f(\mathbf{x})+\frac{\zeta_{1,2}}{2} x_{j} \partial_{j}^{2} f(\mathbf{x})\right\} \quad \text { and } \quad \lambda_{d}=4-4\left(\frac{\sqrt{2}}{\sqrt{3}}\right)^{d}+\left(\frac{1}{\sqrt{2}}\right)^{d} .
$$

Remark 6 The corresponding results when some components are near the boundary $\{0\}$ can be also obtained. For examples, when $\mathbf{x}_{0}=\left(y_{1}, \ldots, y_{d^{\prime}}, \beta \kappa_{d^{\prime}+1}, \ldots, \beta \kappa_{d}\right)^{\prime}$ (its permutation variants are omitted), where $y_{1}, \ldots, y_{d^{\prime}} \in\left(0, c_{U}\right]$ and $\kappa_{d^{\prime}+1}, \ldots, \kappa_{d} \geq 0$ are fixed,

$$
\begin{aligned}
V\left[\widetilde{f}_{\beta, J L N}^{\Pi}\left(\mathbf{x}_{0}\right)\right]= & n^{-1} \beta^{-\left(d-d^{\prime}\right)-d^{\prime} / 2} J\left(\kappa_{d^{\prime}+1}, \ldots, \kappa_{d}\right) \frac{f\left(y_{1}, \ldots, y_{d^{\prime}}, 0, \ldots, 0\right)}{\prod_{j=1}^{d^{\prime}}\left(2 \sqrt{\pi y_{j}}\right)} \\
& +o\left(n^{-1} \beta^{-\left(d-d^{\prime}\right)-d^{\prime} / 2}\right)+O\left(\beta^{5}\right), \quad d^{\prime}=0,1, \ldots, d-1,
\end{aligned}
$$

where

$$
J\left(\kappa_{d^{\prime}+1}, \ldots, \kappa_{d}\right)=4 \prod_{j=d^{\prime}+1}^{d} J_{1}\left(\kappa_{j}\right)-4\left(\frac{\sqrt{2}}{\sqrt{3}}\right)^{d^{\prime}} \prod_{j=d^{\prime}+1}^{d} J_{2}\left(\kappa_{j}\right)+\left(\frac{1}{\sqrt{2}}\right)^{d^{\prime}} \prod_{j=d^{\prime}+1}^{d} J_{3}\left(\kappa_{j}\right) .
$$

The leading term of the variance of $\widetilde{f}_{\beta, J L N}^{\Pi}$ is different from that of the (uncorrected) estimator $\widetilde{f}_{\beta}^{\Pi}$ (actually, for the fixed interior case with $x_{j}>0, j=1, \ldots, d$, the asymptotic variance increases with the factor $\lambda_{d}>1$ ); an incorrect asymptotic variance of Theorem 2.2 in Funke and Kawka (2015) (see also Zougab et al. (2018)) should be corrected so. 
Remark 7 Introduce the subset $\mathcal{D}_{\left[0, c_{U}\right]^{d}}^{\left(d^{\prime}, \tau\right)}=\left\{\mathbf{x} \in\left[0, c_{U}\right]^{d} \mid\right.$ the only $d^{\prime}$ components belong to $\left.\left[0, \beta^{\tau}\right]\right\}$ for $d^{\prime}=1, \ldots, d$. Then, for all sufficiently small $\beta>0, \mathbf{x} \in \mathcal{D}_{\left[0, c_{U}\right]}^{\left(d^{\prime}, \tau\right)}$ implies that

$$
\begin{aligned}
\operatorname{Bias}\left[\widetilde{f}_{\beta, J L N}^{\Pi}(\mathbf{x})\right] & =O\left(\beta^{2 \tau}+n^{-1} \beta^{-d^{\prime}-\left(d-d^{\prime}\right) / 2} \prod_{x_{j} \in\left[\beta^{\tau}, c_{U}\right]} x_{j}^{-1 / 2}\right), \quad \text { if } \tau \in(1 / 2,1), \\
V\left[\widetilde{f}_{\beta, J L N}^{\Pi}(\mathbf{x})\right] & =O\left(n^{-1} \beta^{-d^{\prime}-\left(d-d^{\prime}\right) / 2} \prod_{x_{j} \in\left[\beta^{\tau}, c_{U}\right]} x_{j}^{-1 / 2}+\beta^{5}\right), \quad \text { if } \tau \in[0,1) .
\end{aligned}
$$

As in the univariate case (Subsection 2.2.3), using these bounds, the different rates of the variance and the remainder term of the bias has negligible impact on the MISE (here, the integration is performed in $\left.[0, w]^{d}\right)$. That is, $M I S E_{w}\left[\widetilde{f}_{\beta, J L N}^{\Pi}\right]=A M I S E_{w}\left[\widetilde{f}_{\beta, J L N}^{\Pi}\right]+o\left(\beta^{4}+n^{-1} \beta^{-1 / 2}\right)$, where

$$
A M I S E_{w}\left[\tilde{f}_{\beta, J L N}^{\Pi}\right]=\beta^{4} \int_{[0, w]^{d}} B_{J L N, d}^{2}(\mathbf{x} ; f) d \mathbf{x}+n^{-1} \beta^{-d / 2} \lambda_{d} \int_{[0, w]^{d}} \frac{f(\mathbf{x})}{\prod_{j=1}^{d}\left(2 \sqrt{\pi x_{j}}\right)} d \mathbf{x}
$$

with

$$
B_{J L N, d}(\mathbf{x} ; f)=-f(\mathbf{x}) \gamma_{1, d}\left(\mathbf{x} ; \frac{\gamma_{1, d}(\cdot ; f)}{f(\cdot)}\right) .
$$

Using $\beta \propto n^{-2 /(d+8)}$, which is feasible if $d<8$ (see Assumption ( iii $\left._{d}\right)$ ), the convergence rate $n^{-8 /(d+8)}$ is achieved.

Proof Choosing constants $\tau_{1} \in(4 / 5,1)$ and $\tau_{2} \in(0,1 / 4)$, we have, for all sufficiently small $\beta>0$,

$$
\begin{aligned}
& \int_{\left[\beta^{\tau_{1}}, w\right]^{d}} \operatorname{Bias}^{2}\left[\widetilde{f}_{\beta, J L N}^{\Pi}(\mathbf{x})\right] d \mathbf{x}=\beta^{4} \int_{\left[\beta^{\left.\tau_{1}, w\right]^{d}}\right.} B_{J L N, d}^{2}(\mathbf{x} ; f) d \mathbf{x}+o\left(n^{-1} \beta^{-d / 2}+\beta^{4}\right)+O\left(\left(n^{-1} \beta^{-d / 2}\right)^{2}\{\log (1 / \beta)\}^{d}\right) \\
&=\beta^{4} \int_{[0, w]^{d}} B_{J L N, d}^{2}(\mathbf{x} ; f) d \mathbf{x}+o\left(n^{-1} \beta^{-d / 2}+\beta^{4}\right), \\
& \int_{\left[\beta^{\left.\tau_{2}, w\right]^{d}}\right.} V\left[\widetilde{f}_{\beta, J L N}^{\Pi}(\mathbf{x})\right] d \mathbf{x}=n^{-1} \beta^{-d / 2} \lambda_{d} \int_{\left[\beta^{\tau_{2}}, w\right]^{d}} \frac{f(\mathbf{x})}{\prod_{j=1}^{d}\left(2 \sqrt{\pi x_{j}}\right)} d \mathbf{x}+o\left(n^{-1} \beta^{-d / 2}+\beta^{4}\right) \\
&=n^{-1} \beta^{-d / 2} \lambda_{d} \int_{[0, w]^{d}} \frac{f(\mathbf{x})}{\prod_{j=1}^{d}\left(2 \sqrt{\pi x_{j}}\right)} d \mathbf{x}+o\left(n^{-1} \beta^{-d / 2}+\beta^{4}\right),
\end{aligned}
$$

$\operatorname{and}^{[12]}$

$$
\begin{aligned}
\int_{[0, w]^{d} \backslash\left[\beta^{\left.\tau_{1}, w\right]^{d}}\right.} \operatorname{Bias}^{2}\left[\widetilde{f}_{\beta, J L N}^{\Pi}(\mathbf{x})\right] d \mathbf{x} & =\sum_{d_{L}=1}^{d} O\left(\beta^{4 \tau_{1}+d_{L} \tau_{1}}+\left(n^{-1} \beta^{-d_{L}-\left(d-d_{L}\right) / 2}\right)^{2} \beta^{d_{L} \tau_{1}}\{\log (1 / \beta)\}^{d-d_{L}}\right) \\
& =o\left(n^{-1} \beta^{-d / 2}+\beta^{4}\right),
\end{aligned}
$$

\footnotetext{
${ }^{[12]}$ The subset $[0, w]^{d} \backslash\left[\beta^{\tau_{2}}, w\right]^{d}$ consists of the following two patterns:

(I) For $d_{L}=1, \ldots, d$, the $d_{L}$ components belong to $\left[0, \beta^{\tau_{1}}\right]$, and the remaining $d-d_{L}$ components belong to $\left[\beta^{\tau_{1}}, w\right]$, and

(II) for $d_{M}=1, \ldots, d$, the $d_{M}$ components belong to $\left[\beta^{\tau_{1}}, \beta^{\tau_{2}}\right]$, and the remaining $d-d_{M}$ components belong to $\left[\beta^{\tau_{2}}, w\right]$.

Note that the subset $[0, w]^{d} \backslash\left[\beta^{\tau_{1}}, w\right]^{d}$ consists of the pattern (I) only.
} 


$$
\begin{aligned}
\int_{[0, w]^{d} \backslash\left[\beta^{\left.\tau_{2}, w\right]^{d}}\right.} V\left[\widetilde{f}_{\beta, J L N}^{\Pi}(\mathbf{x})\right] d \mathbf{x}= & \sum_{d_{L}=1}^{d} O\left(n^{-1} \beta^{-d_{L}-\left(d-d_{L}\right) / 2+d_{L} \tau_{1}+\left(d-d_{L}\right) \tau_{1} / 2}+\beta^{5+d_{L} \tau_{1}}\right) \\
& +\sum_{d_{M}=1}^{d} O\left(n^{-1} \beta^{-d / 2+d_{M} \tau_{1} / 2+\left(d-d_{M}\right) \tau_{2} / 2}+\beta^{5+d_{M} \tau_{2}}\right) \\
= & o\left(n^{-1} \beta^{-d / 2}+\beta^{4}\right) .
\end{aligned}
$$

\section{The case $[0,1]^{d}$ : product-type beta $\mathrm{KDE}$}

To estimate a density $f$ with support $[0,1]^{d}$, we now consider the product-type beta KDE, defined by

$$
\widehat{f}_{b}^{(B) \Pi}(x)=\frac{1}{n} \sum_{i=1}^{n} \prod_{j=1}^{d} K^{(B)}\left(X_{i j} ; x_{j}, b\right), \quad \mathbf{x} \in[0,1]^{d}
$$

(we write $K^{(B) \Pi}(\mathbf{s} ; \mathbf{x}, b)=\prod_{j=1}^{d} K^{(B)}\left(s_{j} ; x_{j}, b\right)$ ), where

$$
K^{(B)}(s ; x, b)=\frac{s^{x / b}(1-s)^{(1-x) / b}}{B(x / b+1,(1-x) / b+1)}
$$

is the beta kernel due to Chen (1999). Then, the JLN-type bias-corrected beta KDE is constructed as

$$
\widehat{f}_{b, J L N}^{(B) \Pi}(\mathbf{x})=\left\{\widehat{f}_{b}^{(B) \Pi}(\mathbf{x})+\epsilon\right\} \frac{1}{n} \sum_{i=1}^{n} \frac{K^{(B) \Pi}\left(\mathbf{X}_{i} ; \mathbf{x}, b\right)}{\widehat{f}_{b}^{(B) \Pi}\left(\mathbf{X}_{i}\right)}, \quad \mathbf{x} \in[0,1]^{d} .
$$

Let $\psi(x)=x(1-x)$. Our results in Section 2-4 can be extended, with minor modifications, as follows:

- In addition to $\left(\mathrm{iii}_{d}\right)$, we make the following assumptions:

$\left(\mathrm{i}_{d}^{\sharp}\right)$ A random sample $\left\{\mathbf{X}_{1}, \ldots, \mathbf{X}_{n}\right\}$ is drawn from an unknown density $f$ with support $[0,1]^{d}$.

$\left(\mathrm{ii}_{d}^{\sharp}\right)$ There exists a function $\varphi$, being four times continuously differentiable on $(\underline{c}, \bar{c})^{d}\left(\supset[0,1]^{d}\right)$, such that the target density $f$ is the restriction of $\varphi$ on $[0,1]^{d}$, and there exist constants $\eta_{4} \in(0,1]$ and $L_{4}>0$, such that $\left|\partial_{i_{1}} \partial_{i_{2}} \partial_{i_{3}} \partial_{i_{4}} f(\mathbf{u})-\partial_{i_{1}} \partial_{i_{2}} \partial_{i_{3}} \partial_{i_{4}} f(\mathbf{v})\right| \leq L_{4}\|\mathbf{u}-\mathbf{v}\|^{\eta_{4}}$ for any $\mathbf{u}, \mathbf{v} \in[0,1]^{d}$. $\left(\mathrm{f}_{d}^{\sharp}\right)^{[13]} \inf _{\mathrm{x} \in[0,1]^{d}} f(\mathbf{x})>0$.

- The beta kernel $K^{(B)}(s ; x, b)$ in one dimensional case has no scale parameter, unlike the examples in Section 3; hence, some arguments, relying on Assumption A1, must be re-considered. Also, as variants of the properties in Assumption A2, the beta kernel $K^{(B)}(s ; x, b)$ satisfies:

(i) The $j$ th moments around $x ; \widetilde{\mu}_{j}\left(K^{(B)}(\cdot ; x, b)\right), j=1,2,3,4,6$, are given by

$$
\widetilde{\mu}_{j}\left(K^{(B)}(\cdot ; x, b)\right)=\left\{\begin{array}{rr}
b(1-2 x)-2 b^{2}(1-2 x)+O\left(b^{3}\right), j=1, \\
b x(1-x)+b^{2}\{2-11 x(1-x)\}+O\left(b^{3}\right), j=2, \\
5 b^{2}(1-2 x) x(1-x)+O\left(b^{3}\right), j=3, \\
3 b^{2} x^{2}(1-x)^{2}+O\left(b^{3}\right), j=4, \\
O\left(b^{3}\right), j=6
\end{array}\right.
$$

\footnotetext{
${ }^{[13]}$ This assumption is natural for the compact support case, in which a rigorous treatment of the integrals involving the powers of $1 / f$ is a fairly easy task. On the other hand, for the previous section (the unbounded support case $\left.[0, \infty)^{d}\right)$, we believe that a rather technical assumption (e.g., Assumption A4) is indispensable, due to the unboundedness of $1 / f$.
} 
uniformly in $x \in[0,1]$ (Lemma A.1 in Igarashi (2016a)),

(ii) the mode of the density $K^{(B)}(\cdot ; x, b)$ is given by $x$, where the uniform/nonuniform bounds for $\sup _{s \in[0,1]} K^{(B)}(s ; x, b)=K^{(B)}(x ; x, b)$ are available (Lemma A.3 in Igarashi $\left.(2016 \mathrm{a})\right)^{[14]}$ :

1. $\sup _{x \in[0,1]} K^{(B)}(x ; x, b) \leq b^{-1}(1+b), \quad 2$. whenever $x \in(0,1), K^{(B)}(x ; x, b) \leq \frac{b^{-1 / 2}(1+b)}{\sqrt{2 \pi \psi(x)}}$,

(iii) given constants $\eta \in[0,1)$ and $c_{L}>0$, for all sufficiently small $b>0, x \in\left[c_{L} b^{\eta}, 1-c_{L} b^{\eta}\right]$ implies that

$$
\left|\int_{\mathcal{S}}\left\{K^{(B)}(s ; x, b)\right\}^{2} d s-\frac{1}{2 \sqrt{\pi b \psi(x)}}\right| \leq \frac{M}{\sqrt{b \psi(x)}}\left\{\frac{b}{\psi(x)+b}\right\}
$$

for some constant $M>0$, independent of $b$ and $x$,

(iv) given constants $\eta \in[0,1 / 4)$ and $c_{L}>0$, for all sufficiently small $b>0$, the following approximations hold for $x \in\left[c_{L} b^{\eta}, 1-c_{L} b^{\eta}\right]$ :

$$
\begin{aligned}
\int_{0}^{1} K^{(B)}(t ; x, b) \int_{0}^{1} K^{(B)}(s ; x, b) K^{(B)}(s ; t, b) d s d t & =\frac{1}{\sqrt{6 \pi b \psi(x)}}+o\left(\{b \psi(x)\}^{-1 / 2}\right), \\
\int_{0}^{1} \int_{0}^{1}\left\{\prod_{j=1}^{2} K^{(B)}\left(t_{j} ; x, b\right)\right\} \int_{0}^{1}\left\{\prod_{j=1}^{2} K^{(B)}\left(s ; t_{j}, b\right)\right\} d s d t_{1} d t_{2} & =\frac{1}{2 \sqrt{2 \pi b \psi(x)}}+o\left(\{b \psi(x)\}^{-1 / 2}\right)
\end{aligned}
$$

(as mentioned in Section 3, these calculations can be verified by a Gaussian approximation

$$
\sqrt{b \psi(x)} K^{(B)}\left(x+\sqrt{b \psi(x)} z_{1} ; x+\sqrt{b \psi(x)} z_{2}, b\right)=\phi\left(z_{1}-z_{2}\right)[1+o(1)]
$$

uniformly in $z_{1}, z_{2} \in\left[-b^{\tau} / \sqrt{b \psi(x)}, b^{\tau} / \sqrt{b \psi(x)}\right]$ and $x \in\left[c_{L} b^{\eta}, 1-c_{L} b^{\tau}\right]$; the detail is omitted), and

$\left(\mathrm{iv}^{\prime}\right)$ for all sufficiently small $b>0$, the following approximations hold for $x=b \kappa, 1-b \kappa$ :

$$
\begin{array}{r}
\int_{0}^{1} K^{(B)}(t ; x, b) \int_{0}^{1} K^{(B)}(s ; x, b) K^{(B)}(s ; t, b) d s d t=b^{-1} J_{2}^{(B)}(\kappa)+o\left(b^{-1}\right), \\
\int_{0}^{1} \int_{0}^{1}\left\{\prod_{j=1}^{2} K^{(B)}\left(t_{j} ; x, b\right)\right\} \int_{0}^{1}\left\{\prod_{j=1}^{2} K^{(B)}\left(s ; t_{j}, b\right)\right\} d s d t_{1} d t_{2}=b^{-1} J_{3}^{(B)}(\kappa)+o\left(b^{-1}\right),
\end{array}
$$

where

$$
\begin{aligned}
& J_{2}^{(B)}(\kappa)=\frac{1}{2^{\kappa+1} \Gamma^{2}(\kappa+1)} \int_{0}^{\infty} y^{\kappa} \frac{e^{-y} \Gamma(y+\kappa+1)}{2^{y} \Gamma(y+1)} d y, \\
& J_{3}^{(B)}(\kappa)=\frac{1}{2 \Gamma^{2}(\kappa+1)} \int_{0}^{\infty} \int_{0}^{\infty} y_{1}^{\kappa} y_{2}^{\kappa} \frac{e^{-\left(y_{1}+y_{2}\right)} \Gamma\left(y_{1}+y_{2}+1\right)}{2^{y_{1}+y_{2}} \Gamma\left(y_{1}+1\right) \Gamma\left(y_{2}+1\right)} d y_{1} d y_{2} .
\end{aligned}
$$

${ }^{[14]}$ Whenever $x \in(0,1)$, the density $K^{(B)}(\cdot ; x, b)$ has an exponential small tail as $b \searrow 0$, in the sense that

$$
\begin{aligned}
& \text { 3-1. for } s_{0} \in(0, x], \sup _{0 \leq s \leq s_{0}} K^{(B)}(s ; x, b) \leq b^{-1}(1+b) \exp \left[\frac{1}{b}\left(x \log \frac{s_{0}}{x}+x-s_{0}\right)\right], \\
& 3 \text {-2. for } s_{0}^{\prime} \in[x, 1), \sup _{s_{0}^{\prime} \leq s \leq 1} K^{(B)}(s ; x, b) \leq b^{-1}(1+b) \exp \left[\frac{1}{b}\left\{(1-x) \log \frac{1-s_{0}^{\prime}}{1-x}+(1-x)-\left(1-s_{0}^{\prime}\right)\right\}\right] .
\end{aligned}
$$

The proof is easy, as follows: $K^{(B)}(s ; x, b) / K^{(B)}(x ; x, b)=(s / x)^{x / b}\{(1-s) /(1-x)\}^{(1-x) / b}$ is strictly increasing on $[0, x]$ (strictly decreasing on $[x, 1]$ ); note $\log z-z+1 \leq 0$. 
Note that the above mentioned properties have the $d$-variate counterparts as in Section 4 (the details are omitted to save space).

Now, we define

$$
\gamma_{1, d}^{(B)}(\mathbf{x} ; f)=\sum_{j=1}^{d}\left\{\left(1-2 x_{j}\right) \partial_{j} f(\mathbf{x})+\frac{1}{2} x_{j}\left(1-x_{j}\right) \partial_{j}^{2} f(\mathbf{x})\right\} .
$$

Provided that $\epsilon \propto b^{\iota_{2}}$ for some constant $\iota_{2}>1$, the asymptotic bias and variance at $\mathbf{x}=\left(x_{1}, \ldots, x_{d}\right)^{\prime}$ are given, as follows: For all sufficiently small $b>0$, we have, for $x_{1}, \ldots, x_{d} \in\left[c_{L} b^{\eta}, 1-c_{L} b^{\eta}\right]$,

$$
\begin{aligned}
& \operatorname{Bias}\left[\widehat{f}_{b, J L N}^{(B) \Pi}(\mathbf{x})\right]=b^{2} B_{J L N, d}^{(B)}(\mathbf{x} ; f)+o\left(b^{2}\right)+O\left(n^{-1} b^{-d / 2} \prod_{j=1}^{d}\left\{\psi\left(x_{j}\right)\right\}^{-1 / 2}\right), \quad \text { if } \eta \in[0,1), \\
& V\left[\widehat{f}_{b, J L N}^{(B) \Pi}(\mathbf{x})\right]=n^{-1} b^{-d / 2} \frac{\lambda_{d} f(\mathbf{x})}{\prod_{j=1}^{d} 2 \sqrt{\pi \psi\left(x_{j}\right)}}+o\left(n^{-1} b^{-d / 2} \prod_{j=1}^{d}\left\{\psi\left(x_{j}\right)\right\}^{-1 / 2}\right)+O\left(b^{5}\right), \quad \text { if } \eta \in[0,1 / 4)
\end{aligned}
$$

(note that, if $\eta \in[0,1)$, then, $V\left[\widehat{f}_{b, J L N}^{(B) \Pi}(\mathbf{x})\right]=O\left(n^{-1} b^{-d / 2} \prod_{j=1}^{d}\left\{\psi\left(x_{j}\right)\right\}^{-1 / 2}+b^{5}\right)$ ), where

$$
B_{J L N, d}^{(B)}(\mathbf{x} ; f)=-f(\mathbf{x}) \gamma_{1, d}^{(B)}\left(\mathbf{x} ; \frac{\gamma_{1, d}^{(B)}(\cdot ; f)}{f(\cdot)}\right) .
$$

Remark 8 The corresponding results when some components are near the boundary $\{0,1\}$ can be also obtained. For examples, when $\mathbf{x}_{0}=\left(y_{1}, \ldots, y_{d^{\prime}}, b \kappa_{d^{\prime}+1}, \ldots, b \kappa_{d}\right)^{\prime}$ (its permutation variants are omitted), where, $y_{1}, \ldots, y_{d^{\prime}} \in(0,1)$ and $\kappa_{d^{\prime}+1}, \ldots, \kappa_{d} \geq 0$ are fixed,

$$
\begin{aligned}
V\left[\widehat{f}_{b, J L N}^{(B) \Pi}\left(\mathbf{x}_{0}\right)\right]= & n^{-1} b^{-d^{\prime} / 2-\left(d-d^{\prime}\right)} J^{(B)}\left(\kappa_{d^{\prime}+1}, \ldots, \kappa_{d}\right) \frac{f\left(y_{1}, \ldots, y_{d^{\prime}}, b \kappa_{d^{\prime}+1}, \ldots, b \kappa_{d}\right)}{\prod_{j=1}^{d^{\prime}}\left(2 \sqrt{\pi \psi\left(y_{j}\right)}\right)} \\
& +o\left(n^{-1} b^{-d^{\prime} / 2-\left(d-d^{\prime}\right)}\right)+O\left(b^{5}\right), \quad d^{\prime}=0,1, \ldots, d-1,
\end{aligned}
$$

where

$$
J^{(B)}\left(\kappa_{d^{\prime}+1}, \ldots, \kappa_{d}\right)=4 \prod_{j=d^{\prime}+1}^{d} J_{1}^{(B)}\left(\kappa_{j}\right)-4\left(\frac{\sqrt{2}}{\sqrt{3}}\right)^{d^{\prime}} \prod_{j=d^{\prime}+1}^{d} J_{2}^{(B)}\left(\kappa_{j}\right)+\left(\frac{1}{\sqrt{2}}\right)^{d^{\prime}} \prod_{j=d^{\prime}+1}^{d} J_{3}^{(B)}\left(\kappa_{j}\right) .
$$

Similar results remain valid for $\mathbf{x}_{0}=\left(y_{1}, \ldots, y_{d^{\prime}}, b \kappa_{d^{\prime}+1}, \ldots, b \kappa_{d^{\prime \prime}}, 1-b \kappa_{d^{\prime \prime}+1}, \ldots, 1-b \kappa_{d}\right)^{\prime}$ (and its permutation variants), except that $f$ should be evaluated at $\left(y_{1}, \ldots, y_{d^{\prime}}, 0, \ldots, 0,1, \ldots, 1\right)$.

The leading term of the variance of $\widehat{f}_{b, J L N}^{(B) \Pi}$ is different from that of the (uncorrected) beta KDE

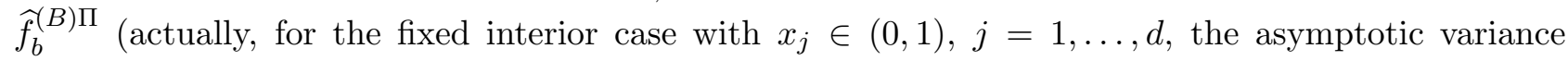
increases with the factor $\lambda_{d}>1$ ); an incorrect asymptotic variance of Theorem 2.2 in Funke and Kawka (2015) (see also Hirukawa (2010)) should be corrected so. Furthermore, the MISE should read as $\int_{[0,1]^{d}} M S E\left[\widehat{f}_{b, J L N}^{(B) \Pi}(\mathbf{x})\right] d \mathbf{x}=A M I S E\left[\widehat{f}_{b, J L N}^{(B) \Pi}\right]+o\left(n^{-1} b^{-d / 2}+b^{4}\right)$, where

$$
A M I S E\left[\widehat{f}_{b, J L N}^{(B) \Pi}\right]=b^{4} \int_{[0,1]^{d}}\left\{B_{J L N, d}^{(B)}(\mathbf{x} ; f)\right\}^{2} d \mathbf{x}+n^{-1} b^{-d / 2} \lambda_{d} \int_{[0,1]^{d}} \frac{f(\mathbf{x})}{\prod_{j=1}^{d}\left(2 \sqrt{\pi \psi\left(x_{j}\right)}\right)} d \mathbf{x}
$$

(the formula given by Funke and Kawka (2015) (see also Hirukawa (2010)) miss the factor $\lambda_{d}$ ). Using $b \propto n^{-2 /(d+8)}$, which is feasible if $d<8$ (see Assumption ( iii $\left._{d}\right)$ ), the convergence rate $n^{-8 /(d+8)}$ is achieved. 
Proof Introducing $\mathcal{D}_{d}^{\left(d^{\prime}, \eta\right)}=\left\{\mathbf{x} \in[0,1]^{d} \mid\right.$ the only $d^{\prime}$ components belong to $\left.\left[0, b^{\eta}\right] \cup\left[1-b^{\eta}, 1\right]\right\}$ for $d^{\prime}=1, \ldots, d$, where $\eta \in[0,1)$ is a constant, we can see that, for all sufficiently small $\beta>0$,

$$
\begin{aligned}
\operatorname{Bias}\left[\widehat{f}_{b, J L N}^{(B) \Pi}(\mathbf{x})\right] & =O\left(b^{2}+n^{-1} b^{-d^{\prime}-\left(d-d^{\prime}\right) / 2} \prod_{x_{j} \in\left[b^{\eta}, 1-b^{\eta}\right]}\left\{\psi\left(x_{j}\right)\right\}^{-1 / 2}\right), \\
V\left[\widehat{f}_{b, J L N}^{(B) \Pi}(\mathbf{x})\right] & =O\left(n^{-1} b^{-d^{\prime}-\left(d-d^{\prime}\right) / 2} \prod_{x_{j} \in\left[b^{\eta}, 1-b^{\eta}\right]}\left\{\psi\left(x_{j}\right)\right\}^{-1 / 2}+b^{5}\right)
\end{aligned}
$$

for $\mathbf{x} \in \mathcal{D}_{d}^{\left(d^{\prime}, \eta\right)}$. Then, choosing constants $\tau_{1} \in(1 / 2,1)$ and $\tau_{2} \in(0,1 / 4)$, we have, for all sufficiently small $b>0$,

$$
\begin{aligned}
& \int_{\left[b^{\left.\tau_{1}, 1-b^{\tau_{1}}\right]^{d}}\right.} \operatorname{Bias}^{2}\left[\widehat{f}_{b, J L N}^{(B) \Pi}(\mathbf{x})\right] d \mathbf{x}=b^{4} \int_{\left[b^{\left.\tau_{1}, 1-b^{\tau_{1}}\right]^{d}}\right.}\left\{B_{J L N, d}^{(B)}(\mathbf{x} ; f)\right\}^{2} d \mathbf{x}+o\left(n^{-1} b^{-d / 2}+b^{4}\right) \\
& +O\left(\left(n^{-1} b^{-d / 2}\right)^{2}\{\log (1 / b)\}^{d}\right) \\
& =b^{4} \int_{[0,1]^{d}}\left\{B_{J L N, d}^{(B)}(\mathbf{x} ; f)\right\}^{2} d \mathbf{x}+o\left(n^{-1} b^{-d / 2}+b^{4}\right), \\
& \int_{\left[b^{\tau_{2}}, 1-b^{\tau_{2}}\right]^{d}} V\left[\widehat{f}_{b, J L N}^{(B) \Pi}(\mathbf{x})\right] d \mathbf{x}=n^{-1} b^{-d / 2} \lambda_{d} \int_{\left[b^{\left.\tau_{2}, 1-b^{\tau_{2}}\right]^{d}}\right.} \frac{f(\mathbf{x})}{\prod_{j=1}^{d}\left(2 \sqrt{\pi \psi\left(x_{j}\right)}\right)} d \mathbf{x}+o\left(n^{-1} b^{-d / 2}+b^{4}\right) \\
& =n^{-1} b^{-d / 2} \lambda_{d} \int_{[0,1]^{d}} \frac{f(\mathbf{x})}{\prod_{j=1}^{d}\left(2 \sqrt{\pi \psi\left(x_{j}\right)}\right)} d \mathbf{x}+o\left(n^{-1} b^{-d / 2}+b^{4}\right),
\end{aligned}
$$

and that ${ }^{[15]}$

$$
\begin{aligned}
\int_{[0,1]^{d} \backslash\left[b^{\tau_{1}}, 1-b^{\tau_{1}}\right]^{d}} \operatorname{Bias}^{2}\left[\widehat{f}_{b, J L N}^{(B) \Pi}(\mathbf{x})\right] d \mathbf{x}= & \sum_{d_{L}=1}^{d} O\left(b^{4+d_{L} \tau_{1}}+\left(n^{-1} b^{-d_{L}-\left(d-d_{L}\right) / 2}\right)^{2} b^{d_{L} \tau_{1}}\{\log (1 / b)\}^{d-d_{L}}\right) \\
= & o\left(n^{-1} b^{-d / 2}+b^{4}\right), \\
\int_{[0,1]^{d} \backslash\left[b^{\left.\tau_{2}, 1-b^{\tau}\right]^{d}}\right.} V\left[\widehat{f}_{b, J L N}^{(B) \Pi}(\mathbf{x})\right] d \mathbf{x}= & \sum_{d_{L}=1}^{d} O\left(n^{-1} b^{-d_{L}-\left(d-d_{L}\right) / 2+d_{L} \tau_{1}+\left(d-d_{L}\right) \tau_{1} / 2}+b^{5+d_{L} \tau_{1}}\right) \\
& +\sum_{d_{M}=1}^{d} O\left(n^{-1} b^{-d / 2+d_{M} \tau_{1} / 2+\left(d-d_{M}\right) \tau_{2} / 2}+b^{5+d_{M} \tau_{2}}\right) \\
= & o\left(n^{-1} b^{-d / 2}+b^{4}\right) .
\end{aligned}
$$

\section{Simulation studies}

We illustrate, through the simulations, the finite sample performance of the JLN-type bias-corrected Amoroso/IG/BS/RIG/LN[-1/2] KDEs, together with their uncorrected estimators (Examples 1-3).

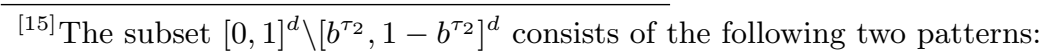

(I) For $d_{L}=1, \ldots, d$, the $d_{L}$ components belong to $\left[0, b^{\tau_{1}}\right] \bigcup\left[1-b^{\tau_{1}}, 1\right]$, and the remaining $d-d_{L}$ components belong to $\left[b^{\tau_{1}}, 1-b^{\tau_{1}}\right]$, and

(II) for $d_{M}=1, \ldots, d$, the $d_{M}$ components belong to $\left[b^{\tau_{1}}, b^{\tau_{2}}\right] \bigcup\left[1-b^{\tau_{2}}, 1-b^{\tau_{1}}\right]$, and the remaining $d-d_{M}$ components belong to $\left[b^{\tau_{2}}, 1-b^{\tau_{2}}\right]$.

Note that the subset $[0,1]^{d} \backslash\left[b^{\tau_{1}}, 1-b^{\tau_{1}}\right]^{d}$ consists of the pattern (I) only.
} 
We generated 1000 replicate samples of $n=100,300$ from the five densities:
A. $f(x)=\frac{1}{2}\left(\frac{e^{-x / 3}}{3}+\frac{x e^{-x / 3}}{9}\right)$
B. $f(x)=\frac{e^{-x / 3}}{3}$,
C. $f(x)=\frac{1}{2}\left(\frac{e^{-x / 10}}{10}+x e^{-x}\right)$,
D. $f(x)=\frac{1}{2}\left[\frac{1}{\sqrt{2 \pi} 0.8 x} \exp \left\{-\frac{(\log x-1)^{2}}{2(0.8)^{2}}\right\}+\frac{1}{\sqrt{2 \pi} 0.4 x} \exp \left\{-\frac{(\log x-2)^{2}}{2(0.4)^{2}}\right\}\right]$,
E. $f(x)=\frac{2}{\sqrt{2 \pi}} \exp \left(-\frac{x^{2}}{2}\right)$,

and calculated the integrated squared error (ISE); ISE $E_{k}=\int_{0}^{\infty}\left\{\widetilde{f}^{[k]}(x)-f(x)\right\}^{2} d x$ for the $k$ th sample. Each smoothing parameter $b$ was chosen using the least squared cross-validation method (here, we chose $\left.\epsilon=0.000001 \times b^{2}\right)$. Tables $1-5$ show that the average ISEs; $(1 / 1000) \sum_{k=1}^{1000} I S E_{k}$ decreased, as the sample size $n$ increased. Overall, the JLN-type bias-corrected KDEs $\widehat{f}_{b, J L N}^{(\#)}$, using the linear $\rho$-function; $\rho(t)=t+1$, outperformed the uncorrected estimators $\widehat{f}_{b}^{(\#)}$, except for cases $\mathrm{C}$ and $\mathrm{D}$ $(n=100)$. As expected, when the shoulder condition $f^{(1)}(0)=0$ is satisfied (cases $\mathrm{A}$ and $\left.\mathrm{E}\right)$, the two-regime version, denoted by $\widetilde{f}_{b, J L N}^{\star(\#)}$, using $r_{1 / 4}(t)=(5 / 4)(t / 2)^{8 / 5}+1$ (for the Amoroso case, $\left.r_{1 /(4|\gamma|)}(t)=\{1 /(4|\gamma|)+1\}(t / 2)^{2 /(1 /(4|\gamma|)+1)}+1\right)$; see Subsection 3.2, also worked well, whereas, for cases B and $\mathrm{C}$, having $f^{(1)}(0) \neq 0, \widetilde{f}_{b, J L N}^{\star(\#)}$ almost behaved worse than $\widehat{f}_{b, J L N}^{(\#)}$. Additionally, we compared the JLN-type with other bias corrections (see Igarashi and Kakizawa (2018b)), reviewed in Introduction. We observe from Table 6 ; the simulation results $(n=300)$ of the $\mathrm{SS}_{1}$-type and $\mathrm{JF}_{1}$-type bias-corrected Amoroso KDEs, that, for case B, the JLN-type outperformed the $\mathrm{SS}_{1}$-type and $\mathrm{JF}_{1}$-type. On the other hand, for cases $\mathrm{A}$ and $\mathrm{E}$, since the shoulder condition $f^{(1)}(0)=0$ is satisfied, the two-regime JLN-type worked very well. For cases $\mathrm{C}$ and D, it may be difficult to decide whether the JLN-type was superior or inferior to the $\mathrm{SS}_{1}$-type and $\mathrm{JF}_{1}$-type, since the numerical results contradicted with the graph of the AMISE asymptotic efficiency; this may be caused by the small sample size $n$. In summary, our simulation results confirm the bias reduction. It is worthwhile to note that the two-regime version, especially, the choice of $c=1 / 4(c=1 /(4|\gamma|)$ for the Amoroso case), had the advantage over the linear $\rho$-function, i.e., the average ISEs of $\widetilde{f}_{b}^{(\#)}$ were almost smaller than those of $\widehat{f}_{b}^{(\#)}$. However, the present simulation results indicate that, without the shoulder condition $f^{(1)}(0)=0$, such a two-regime formulation is incompatible with the bias correction; this was already pointed out by Igarashi and Kakizawa (2015, 2018a) for the SS/TS/JF-types.

\section{Appendix}

For simplicity, we write $\zeta_{\beta}(x)=\widetilde{f}_{\beta}(x)-f(x)+\epsilon$, and

$$
U_{x, \beta, i}=\frac{f(x)+\zeta_{\beta}(x)}{f\left(X_{i}\right)+\zeta_{\beta}\left(X_{i}\right)} K\left(X_{i} ; x, \beta\right), \quad i=1, \ldots, n .
$$

Then,

$$
E\left[\widetilde{f}_{\beta, J L N}(x)\right]=\frac{1}{n} \sum_{i=1}^{n} E\left[U_{x, \beta, i}\right]=E\left[U_{x, \beta, 1}\right],
$$




$$
V\left[\widetilde{f}_{\beta, J L N}(x)\right]=\frac{1}{n^{2}} \sum_{i, j=1}^{n} \operatorname{Cov}\left[U_{x, \beta, i}, U_{x, \beta, j}\right]=\frac{1}{n} V\left[U_{x, \beta, 1}\right]+\frac{n-1}{n} \operatorname{Cov}\left[U_{x, \beta, 1}, U_{x, \beta, 2}\right],
$$

where, by virtue of the law of total variance/covariance,

$$
\begin{aligned}
V\left[U_{x, \beta, 1}\right] & =E\left[V\left[U_{x, \beta, 1} \mid X_{1}\right]\right]+V\left[E\left[U_{x, \beta, 1} \mid X_{1}\right]\right] \\
\operatorname{Cov}\left[U_{x, \beta, 1}, U_{x, \beta, 2}\right] & =E\left[\operatorname{Cov}\left[U_{x, \beta, 1}, U_{x, \beta, 2} \mid X_{1}, X_{2}\right]\right]+\operatorname{Cov}\left[E\left[U_{x, \beta, 1} \mid X_{1}, X_{2}\right], E\left[U_{x, \beta, 2} \mid X_{1}, X_{2}\right]\right] .
\end{aligned}
$$

Define, for $\ell=1,2$,

$$
\mathcal{P}_{\ell}^{[0]}(x)=\frac{f(x)}{f\left(X_{\ell}\right)} \quad \text { and } \quad \mathcal{P}_{\ell}^{[j]}(x)=-\zeta_{\beta}(x) \zeta_{\beta}^{j-1}\left(X_{\ell}\right)+\frac{f(x) \zeta_{\beta}^{j}\left(X_{\ell}\right)}{f\left(X_{\ell}\right)}, \quad j=1,2, \ldots
$$

We use the stochastic expansion

$$
\frac{f(x)+\zeta_{\beta}(x)}{f\left(X_{\ell}\right)+\zeta_{\beta}\left(X_{\ell}\right)}=\sum_{j=0}^{m}(-1)^{j} \frac{\mathcal{P}_{\ell}^{[j]}(x)}{f^{j}\left(X_{\ell}\right)}+\mathcal{R}_{[m], \ell}^{(J L N)}(x) \quad \text { for } m=0,2,4 .
$$

To complete the proofs below, we must deal with the integrals involving the unbounded function of the power of $1 / f$, as well as the asymptotic negligibility of the remainder term $\mathcal{R}_{[m], \ell}^{(J L N)}(x)$. Details are in supplemental issue: Supplemental appendix to "Multiplicative bias correction for asymmetric kernel density estimators revisited", Faculty of Economics, Hokkaido University, Discussion Paper Series A: No. 2018-328.

Proof of Proposition 1 Using the stochastic expansion (we set $m=2$ ), we have

$$
\begin{aligned}
E\left[U_{x, \beta, 1}\right]-f(x)= & E\left[\frac{K\left(X_{1} ; x, \beta\right)}{f\left(X_{1}\right)} E\left[\zeta_{\beta}(x) \mid X_{1}\right]\right]-f(x) E\left[\frac{K\left(X_{1} ; x, \beta\right)}{f^{2}\left(X_{1}\right)} E\left[\zeta_{\beta}\left(X_{1}\right) \mid X_{1}\right]\right] \\
& -E\left[\frac{K\left(X_{1} ; x, \beta\right)}{f^{2}\left(X_{1}\right)} E\left[\zeta_{\beta}(x) \zeta_{\beta}\left(X_{1}\right) \mid X_{1}\right]\right]+f(x) E\left[\frac{K\left(X_{1} ; x, \beta\right)}{f^{3}\left(X_{1}\right)} E\left[\zeta_{\beta}^{2}\left(X_{1}\right) \mid X_{1}\right]\right] \\
& +E\left[K\left(X_{1} ; x, \beta\right) E\left[\mathcal{R}_{[2], 1}^{(J L N)}(x) \mid X_{1}\right]\right] \\
= & I_{1,1}(x)-f(x) I_{1,2}(x)-I_{1,3}(x)+f(x) I_{1,4}(x)+I_{1,5}(x) \quad \text { (say). }
\end{aligned}
$$

It is shown that, given constants $\eta \in[0,1)$ and $0<c_{L}<c_{R}$, for all sufficiently small $\beta>0$,

$$
\begin{aligned}
& I_{1,1}(x)-\left\{\epsilon+B_{\beta}^{(K), f}(x)\right\}=\left\{\begin{array}{l}
O\left(n^{-1}(\beta x)^{-1 / 2}\right) \text { for } x \in\left[c_{L} \beta^{\eta}, c_{U}\right], \\
O\left(n^{-1} \beta^{-1}\right) \text { for } x=\beta \kappa,
\end{array}\right. \\
& I_{1,2}(x)-\int_{\mathcal{S}} \frac{K(s ; x, \beta)}{f(s)}\left\{\epsilon+B_{\beta}^{(K), f}(s)\right\} d s=\left\{\begin{array}{l}
O\left(n^{-1}(\beta x)^{-1 / 2}\right) \quad \text { for } x \in\left[c_{L} \beta^{\eta}, c_{U}\right], \\
O\left(n^{-1} \beta^{-1}\right) \text { for } x=\beta \kappa,
\end{array}\right. \\
& I_{1,3}(x)-B_{\beta}^{(K), f}(x) \int_{\mathcal{S}} \frac{K(s ; x, \beta)}{f(s)} B_{\beta}^{(K), f}(s) d s=\left\{\begin{array}{l}
o\left(\beta^{2}\right)+O\left(n^{-1}(\beta x)^{-1 / 2}\right) \quad \text { for } x \in\left[c_{L} \beta^{\eta}, c_{U}\right], \\
o\left(\beta^{2}\right)+O\left(n^{-1} \beta^{-1}\right) \quad \text { for } x=\beta \kappa,
\end{array}\right. \\
& I_{1,4}(x)-\int_{\mathcal{S}} \frac{K(s ; x, \beta)}{f^{2}(s)}\left\{B_{\beta}^{(K), f}(s)\right\}^{2} d s=\left\{\begin{array}{l}
o\left(\beta^{2}\right)+O\left(n^{-1}(\beta x)^{-1 / 2}\right) \quad \text { for } x \in\left[c_{L} \beta^{\eta}, c_{U}\right], \\
o\left(\beta^{2}\right)+O\left(n^{-1} \beta^{-1}\right) \text { for } x=\beta \kappa,
\end{array}\right. \\
& I_{1,5}(x)=\left\{\begin{array}{l}
o\left(\beta^{2}+n^{-1}(\beta x)^{-1 / 2}\right) \quad \text { for } x \in\left[c_{L} \beta^{\eta}, c_{U}\right] \\
o\left(\beta^{2}+n^{-1} \beta^{-1}\right) \quad \text { for } x=\beta \kappa
\end{array}\right.
\end{aligned}
$$


The result follows from

$$
\begin{aligned}
& I_{1,1}(x)-f(x) I_{1,2}(x)=\left\{\begin{array}{l}
-\beta^{2} f(x) \gamma_{1}\left(x ; \frac{\gamma_{1}(\cdot ; f)}{f(\cdot)}\right)+o\left(\beta^{2}\right)+O\left(n^{-1}(\beta x)^{-1 / 2}\right) \quad \text { for } x \in\left[c_{L} \beta^{\eta}, c_{U}\right], \\
\beta^{2}\left[\left\{\mu_{1}^{\prime}(0)\right\}^{2} \frac{\left\{f^{(1)}(0)\right\}^{2}}{f(0)}+\left\{-\frac{1}{2} \int_{\mathcal{S}} \mu_{2}^{\prime}(y) p_{K}(y ; \kappa) d y+\mu_{2}^{\prime}(\kappa)-\frac{\kappa^{2}}{2}\right\} f^{(2)}(0)\right] \\
+o\left(\beta^{2}\right)+O\left(n^{-1} \beta^{-1}\right) \quad \text { for } x=\beta \kappa,
\end{array}\right. \\
& I_{1,3}(x)-f(x) I_{1,4}(x)=\left\{\begin{array}{c}
o\left(\beta^{2}\right)+O\left(n^{-1}(\beta x)^{-1 / 2}\right) \quad \text { for } x \in\left[c_{L} \beta^{\eta}, c_{U}\right], \\
o\left(\beta^{2}\right)+O\left(n^{-1} \beta^{-1}\right) \text { for } x=\beta \kappa,
\end{array}\right.
\end{aligned}
$$

noting that $(7),(8)$,

$$
\begin{aligned}
& \int_{\mathcal{S}} \frac{K(s ; x, \beta)}{f(s)} B_{\beta}^{(K), f}(s) d s=\left\{\begin{array}{l}
\beta \frac{\gamma_{1}(x ; f)}{f(x)}+\beta^{2}\left\{\frac{\gamma_{2}(x ; f)}{f(x)}+\gamma_{1}\left(x ; \frac{\gamma_{1}(\cdot ; f)}{f(\cdot)}\right)\right\}+o\left(\beta^{2}\right) \quad \text { for } x \in\left[c_{L} \beta^{\eta}, c_{U}\right], \\
\beta \mu_{1}^{\prime}(0) \frac{f^{(1)}(0)}{f(0)}+\beta^{2}\left[-\mu_{1}^{\prime}(0)\left\{\mu_{1}^{\prime}(0)+\kappa\right\}\left\{\frac{f^{(1)}(0)}{f(0)}\right\}^{2}\right. \\
\left.\quad+\frac{1}{2}\left\{\int_{\mathcal{S}} \mu_{2}^{\prime}(y) p_{K}(y ; \kappa) d y-\mu_{2}^{\prime}(\kappa)\right\} \frac{f^{(2)}(0)}{f(0)}\right]+O\left(\beta^{3}\right) \quad \text { for } x=\beta \kappa,
\end{array}\right. \\
& \int_{\mathcal{S}} \frac{K(s ; x, \beta)}{f^{2}(s)}\left\{B_{\beta}^{(K), f}(s)\right\}^{2} d s=\left\{\begin{array}{c}
\beta^{2}\left\{\frac{\gamma_{1}(x ; f)}{f(x)}\right\}^{2}+o\left(\beta^{2}\right) \text { for } x \in\left[c_{L} \beta^{\eta}, c_{U}\right], \\
\beta^{2}\left\{\mu_{1}^{\prime}(0)\right\}^{2}\left\{\frac{f^{(1)}(0)}{f(0)}\right\}^{2}+O\left(\beta^{3}\right) \text { for } x=\beta \kappa,
\end{array}\right.
\end{aligned}
$$$$
\sup _{x \in\left[0, c_{U}\right]}\left|\int_{\mathcal{S}} \frac{K(s ; x, \beta)}{f(s)} d s-\frac{1}{f(x)}\right|=O(\beta) .
$$

Remark A.1 The vanishing of the $O\left(\beta^{j}\right)$-terms in $I_{1,2 j-1}(x)$ and $I_{1,2 j}(x)$, for $j=1,2$ and $x=\beta \kappa$, is ensured by Assumption A1.3. Actually, if Assumption A1.3 is replaced by A1.3 $3^{\sharp}$, then, for $x=\beta \kappa$,

$$
\begin{aligned}
& I_{1,1}(x)-f(x) I_{1,2}(x) \\
& =\beta\left[\left\{\mu_{1}^{\prime}(\kappa)-\kappa\right\}-\int_{\mathcal{S}}\left\{\mu_{1}^{\prime}(y)-y\right\} p_{K}(y ; \kappa) d y\right] f^{(1)}(0) \\
& \quad+\beta^{2}\left[\int_{\mathcal{S}}(y-\kappa)\left\{\mu_{1}^{\prime}(y)-y\right\} p_{K}(y ; \kappa) d y \frac{\left\{f^{(1)}(0)\right\}^{2}}{f(0)}+\left\{-\frac{1}{2} \int_{\mathcal{S}} \mu_{2}^{\prime}(y) p_{K}(y ; \kappa) d y+\mu_{2}^{\prime}(\kappa)-\frac{\kappa^{2}}{2}\right\} f^{(2)}(0)\right] \\
& \quad+o\left(\beta^{2}\right)+O\left(n^{-1} \beta^{-1}\right), \\
& I_{1,3}(x)-f(x) I_{1,4}(x) \\
& =\beta^{2}\left[\left\{\mu_{1}^{\prime}(\kappa)-\kappa\right\} \int_{\mathcal{S}}\left\{\mu_{1}^{\prime}(y)-y\right\} p_{K}(y ; \kappa) d y-\int_{\mathcal{S}}\left\{\mu_{1}^{\prime}(y)-y\right\}^{2} p_{K}(y ; \kappa) d y\right] \frac{\left\{f^{(1)}(0)\right\}^{2}}{f(0)} \\
& \quad+o\left(\beta^{2}\right)+O\left(n^{-1} \beta^{-1}\right) .
\end{aligned}
$$

Proof of Proposition 2 It is easy to see that, given constants $\eta \in[0,1)$ and $0<c_{L}<c_{R}$, for all sufficiently small $\beta>0$,

$$
\begin{aligned}
\frac{1}{n} E\left[V\left[U_{x, \beta, 1} \mid X_{1}\right]\right] & \leq \frac{1}{n}\left\{\sup _{s \in \mathcal{S}} K(s ; x, \beta)\right\} E\left[K\left(X_{1} ; x, \beta\right) E\left[\left\{\mathcal{R}_{[0], 1}^{(J L N)}(x)\right\}^{2} \mid X_{1}\right]\right] \\
& =\left\{\begin{array}{l}
o\left(n^{-1}(\beta x)^{-1 / 2}\right) \quad \text { for } x \in\left[c_{L} \beta^{\eta}, c_{U}\right] \\
o\left(n^{-1} \beta^{-1}\right) \text { for } x=\beta \kappa .
\end{array}\right.
\end{aligned}
$$


Using the stochastic expansion $(m=0,2,4)$, it can be shown that, for all sufficiently small $\beta>0$,

$$
\begin{aligned}
\frac{1}{n} V\left[E\left[U_{x, \beta, 1} \mid X_{1}\right]\right]-\frac{1}{n} \mathcal{I}_{1}(x)=\left\{\begin{array}{l}
o\left(n^{-1}(\beta x)^{-1 / 2}\right) \text { for } x \in\left[c_{L} \beta^{\eta}, c_{U}\right], \\
o\left(n^{-1} \beta^{-1}\right) \text { for } x=\beta \kappa,
\end{array}\right. \\
E\left[\operatorname{Cov}\left[U_{x, \beta, 1}, U_{x, \beta, 2} \mid X_{1}, X_{2}\right]\right]-\frac{1}{n} \mathcal{I}_{2}(x)=\left\{\begin{array}{l}
o\left(n^{-1}(\beta x)^{-1 / 2}\right)+O\left(\beta^{5}\right) \text { for } x \in\left[c_{L} \beta^{\eta}, c_{U}\right], \\
o\left(n^{-1} \beta^{-1}\right)+O\left(\beta^{5}\right) \text { for } x=\beta \kappa,
\end{array}\right. \\
\operatorname{Cov}\left[E\left[U_{x, \beta, 1} \mid X_{1}, X_{2}\right], E\left[U_{x, \beta, 2} \mid X_{1}, X_{2}\right]-\frac{1}{n} \mathcal{I}_{3}(x)= \begin{cases}o\left(n^{-1}(\beta x)^{-1 / 2}\right)+O\left(\beta^{5}\right) & \text { for } x \in\left[c_{L} \beta^{\eta}, c_{U}\right], \\
o\left(n^{-1} \beta^{-1}\right)+O\left(\beta^{5}\right) & \text { for } x=\beta \kappa,\end{cases} \right.
\end{aligned}
$$

where

$$
\begin{aligned}
& \mathcal{I}_{1}(x)=f^{2}(x) \int_{\mathcal{S}} \frac{K^{2}(s ; x, \beta)}{f(s)} d s, \\
& \mathcal{I}_{2}(x)=\int_{\mathcal{S}} K^{2}(s ; x, \beta) f(s) d s-2 f(x) \int_{\mathcal{S}} \frac{K(t ; x, \beta)}{f(t)} \int_{\mathcal{S}} K(s ; x, \beta) K(s ; t, \beta) f(s) d s d t \\
& +f^{2}(x) \int_{\mathcal{S}} \int_{\mathcal{S}} \frac{K(t ; x, \beta)}{f(t)} \frac{K(u ; x, \beta)}{f(u)} \int_{\mathcal{S}} K(s ; t, \beta) K(s ; u, \beta) f(s) d s d t d u, \\
& \mathcal{I}_{3}(x)=2 f(x) \int_{\mathcal{S}} K^{2}(s ; x, \beta) d s-2 f^{2}(x) \int_{\mathcal{S}} \frac{K(t ; x, \beta)}{f(t)} \int_{\mathcal{S}} K(s ; x, \beta) K(s ; t, \beta) d s d t
\end{aligned}
$$

(note that $n^{-1} \sum_{j=1}^{3} \mathcal{I}_{j}(x)=O\left(n^{-1}(\beta x)^{-1 / 2}\right)$ for $x \in\left[c_{L} \beta^{\eta}, c_{U}\right]$, if $\eta \in[0,1)$ ). The result follows from

$$
\begin{aligned}
& n^{-1} \sum_{j=1}^{3} \mathcal{I}_{j}(x) \\
& = \begin{cases}n^{-1} \beta^{-1 / 2}\left(4-4 \frac{\sqrt{2}}{\sqrt{3}}+\frac{1}{\sqrt{2}}\right) \frac{f(x)}{2 \sqrt{\pi x}}+o\left(n^{-1}(\beta x)^{-1 / 2}\right) & \text { for } x \in\left[c_{L} \beta^{\eta}, c_{U}\right], \text { if } \eta \in[0,1 / 4), \\
n^{-1} \beta^{-1}\left\{4 J_{1}(\kappa)-4 J_{2}(\kappa)+J_{3}(\kappa)\right\} f(0)+o\left(n^{-1} \beta^{-1}\right) & \text { for } x=\beta \kappa .\end{cases}
\end{aligned}
$$

\section{References}

Chen, S. X. (1999) "Beta kernel estimators for density functions", Computational Statistics and Data Analysis, 31, 131-145.

Chen, S. X. (2000) "Probability density function estimation using gamma kernels", Annals of the Institute of Statistical Mathematics, 52, 471-480.

Chen, S.-M., Hsu, Y.-S. and Liaw, J.-T. (2009) "On kernel estimators of density ratio", Statistics, 43, 463-479.

Funke, B. and Kawka, R. (2015) "Nonparametric density estimation for multivariate bounded data using two non-negative multiplicative bias correction methods", Computational Statistics and Data Analysis, 92, 148-162.

Hagmann, M. and Scaillet, O. (2007) "Local multiplicative bias correction for asymmetric kernel density estimators", Journal of Econometrics, 141, 213-249.

Hirukawa, M. (2010) "Nonparametric multiplicative bias correction for kernel-type density estimation on the unit interval", Computational Statistics and Data Analysis, 54, 473-495. Correction: (2016), 95, 240-242. 
Hirukawa, M. and Sakudo, M. (2014) "Nonnegative bias reduction methods for density estimation using asymmetric kernels", Computational Statistics and Data Analysis, 75, 112-123.

Hirukawa, M. and Sakudo, M. (2015) "Family of the generalised gamma kernels: a generator of asymmetric kernels for nonnegative data", Journal of Nonparametric Statistics, 27, 41-63.

Igarashi, G. (2016a) "Bias reductions for beta kernel estimation", Journal of Nonparametric Statistics, $28,1-30$.

Igarashi, G. (2016b) "Weighted log-normal kernel density estimation", Communications in Statistics - Theory and Methods, 45, 6670-6687.

Igarashi, G. and Kakizawa, Y. (2014a) "On improving convergence rate of Bernstein polynomial density estimator", Journal of Nonparametric Statistics, 26, 61-84.

Igarashi, G. and Kakizawa, Y. (2014b) "Re-formulation of inverse Gaussian, reciprocal inverse Gaussian, and Birnbaum-Saunders kernel estimators", Statistics and Probability Letters, 84, 235-246.

Igarashi, G. and Kakizawa, Y. (2015) "Bias corrections for some asymmetric kernel estimators", Journal of Statistical Planning and Inference, 159, 37-63.

Igarashi, G. and Kakizawa, Y. (2018a) "Generalised gamma kernel density estimation for nonnegative data and its bias reduction", To appear in Journal of Nonparametric Statistics.

Igarashi, G. and Kakizawa, Y. (2018b) "Limiting bias-reduced Amoroso kernel density estimators for nonnegative data", Communications in Statistics - Theory and Methods, 47, 4905-4937.

Jin, X. and Kawczak, J. (2003) "Birnbaum-Saunders and lognormal kernel estimators for modelling durations in high frequency financial data", Annals of Economics and Finance, 4, 103-124.

Jones, M. C. (1993) "Simple boundary correction for kernel density estimation", Statistics and Computing, 3, 135-146.

Jones, M. C. and Foster, P. J. (1993) "Generalized jackknifing and higher order kernels", Journal of Nonparametric Statistics, 3, 81-94.

Jones, M. C., Linton, O. and Nielsen, J. P. (1995) "A simple bias reduction method for density estimation", Biometrika, 82, 327-338.

Kakizawa, Y. (2018) "Nonparametric density estimation for nonnegative data, using symmetricalbased inverse and reciprocal inverse Gaussian kernels through dual transformation", Journal of Statistical Planning and Inference, 193, 117-135.

Koul, H. L. and Song, W. (2013) "Large sample results for varying kernel regression estimates", Journal of Nonparametric Statistics, 25, 829-853.

Leblanc, A. (2010) "A bias-reduced approach to density estimation using Bernstein polynomials", Journal of Nonparametric Statistics, 22, 459-475.

Marchant, C., Bertin, K., Leiva, V. and Saulo, H. (2013) "Generalized Birnbaum-Saunders kernel density estimators and an analysis of financial data", Computational Statistics and Data Analysis, $63,1-15$.

Marron, J. S. and Ruppert, D. (1994) "Transformations to reduce boundary bias in kernel density estimation", Journal of the Royal Statistical Society, Series B, 56, 653-671.

Rosenblatt, M. (1956) "Remarks on some nonparametric estimates of a density function", The Annals of Mathematical Statistics, 27, 832-837. 
Saulo, H., Leiva, V., Ziegelmann, F. A. and Marchant, C. (2013) "A nonparametric method for estimating asymmetric densities based on skewed Birnbaum-Saunders distributions applied to environmental data", Stochastic Environmental Research and Risk Assessment, 27, 1479-1491.

Scaillet, O. (2004) "Density estimation using inverse and reciprocal inverse Gaussian kernels", Journal of Nonparametric Statistics, 16, 217-226.

Schucany, W. R. and Sommers, J. P. (1977) "Improvement of kernel type density estimators", Journal of the American Statistical Association, 72, 420-423.

Silverman, B. W. (1986) Density Estimation for Statistics and Data Analysis, London: Chapman \& Hall.

Terrell, G. R. and Scott, D. W. (1980) "On improving convergence rates for nonnegative kernel density estimators", The Annals of Statistics, 8, 1160-1163.

Wand, M. P. and Jones, M. C. (1995) Kernel Smoothing, London: Chapman \& Hall.

Wand, M. P. and Schucany, W. R. (1990) "Gaussian-based kernels", Canadian Journal of Statistics, $18,197-204$.

Zhang, S., Karunamuni, R. J. and Jones, M. C. (1999) "An improved estimator of the density function at the boundary", Journal of the American Statistical Association, 94, 1231-1241.

Zougab, N. and Adjabi, S. (2016) "Multiplicative bias correction for generalized Birnbaum-Saunders kernel density estimators and application to nonnegative heavy tailed data", Journal of the Korean Statistical Society, 45, 51-63.

Zougab, N., Harfouche, L., Ziane, Y. and Adjabi, S. (2018) "Multivariate generalized BirnbaumSaunders kernel density estimators", Communications in Statistics - Theory and Methods, 47, 45344555 . 
Table 1: Case A. The average ISEs $\times 10^{6}$.

The number in the parentheses stands for the standard deviation $\times 10^{6}$ of the ISEs.

\begin{tabular}{|c|c|c|c|c|c|c|c|c|c|c|c|c|c|}
\hline$n$ & linear & $A_{2}$ & $A_{1.5}$ & $A_{1}$ & $A_{0.5}$ & $A_{-0.5}$ & $A_{-1}$ & $A_{-1.5}$ & $A_{-2}$ & $I G$ & $B S$ & $R I G$ & $L N[-1 / 2]$ \\
\hline \multirow[t]{4}{*}{100} & $\widehat{f}_{b}^{(\#)}$ & 3389 & 3263 & 3006 & 2914 & 3201 & 3392 & 3735 & 4135 & 3316 & 3539 & 3751 & 3288 \\
\hline & & $(2946)$ & $(3216)$ & $(3144)$ & $(3206)$ & (3235) & $(2898)$ & $(2674)$ & (2941) & $(3346)$ & (3411) & (3468) & $(3317)$ \\
\hline & $\widehat{f}_{b, J L N}^{(\#)}$ & 2105 & 2255 & 2239 & 2497 & 2639 & 2763 & 3160 & 3583 & 2482 & 2569 & 2591 & 2515 \\
\hline & & (2403) & $(2781)$ & $(2673)$ & $(2738)$ & $(2271)$ & $(2424)$ & $(2746)$ & $(3035)$ & $(2591)$ & $(2936)$ & (2996) & $(2741)$ \\
\hline \multirow[t]{4}{*}{300} & $\widehat{f}_{b}^{(\#)}$ & 1483 & 1399 & 1240 & 1193 & 1252 & 1450 & 1633 & 1772 & 1356 & 1460 & 1615 & 1358 \\
\hline & & (1172) & (1269) & $(1166)$ & (1485) & (1142) & $(1220)$ & (1154) & (1088) & $(1280)$ & (1287) & (1351) & (1279) \\
\hline & $\widehat{f}_{b, J L N}^{(\#)}$ & 888 & 895 & 909 & 1003 & 1086 & 1099 & 1237 & 1379 & 990 & 1017 & 1061 & 995 \\
\hline & & $(887)$ & $(989)$ & (1127) & (1329) & (1068) & $(897)$ & (935) & (1030) & $(905)$ & (999) & $(985)$ & $(936)$ \\
\hline$n$ & two-regime & $A_{2}$ & $A_{1.5}$ & $A_{1}$ & $A_{0.5}$ & $A_{-0.5}$ & $A_{-1}$ & $A_{-1.5}$ & $A_{-2}$ & $I G$ & $B S$ & $R I G$ & $L N[-1 / 2]$ \\
\hline \multirow[t]{4}{*}{100} & $\tilde{f}_{b}^{\star}(\#)$ & 2422 & 2479 & 2596 & 2979 & 3258 & 2920 & 2799 & 2850 & 2710 & 2768 & 2650 & 2698 \\
\hline & & $(2825)$ & $(2881)$ & $(2997)$ & $(3058)$ & (2921) & $(2807)$ & $(2537)$ & $(2571)$ & $(2772)$ & $(2896)$ & $(2764)$ & (2806) \\
\hline & $\begin{array}{l}\tilde{f}_{b, J L N}^{\star(\#)} \\
\text { (\#) }\end{array}$ & 2025 & 2017 & 2000 & 2258 & 2401 & 2305 & 2417 & 2581 & 2189 & 2434 & 2565 & 2146 \\
\hline & & $(2795)$ & $(2798)$ & $(2803)$ & $(2616)$ & $(2604)$ & $(2386)$ & $(2412)$ & $(2512)$ & $(2706)$ & $(2759)$ & $(2724)$ & $(2637)$ \\
\hline \multirow[t]{4}{*}{300} & $\tilde{f}_{b}^{\star}(\#)$ & 931 & 961 & 1032 & 1193 & 1332 & 1154 & 1095 & 1100 & 1104 & 1084 & 1034 & 1082 \\
\hline & & (1059) & $(1105)$ & $(1120)$ & (1138) & (1236) & $(1032)$ & (919) & $(935)$ & (1121) & (1174) & (1035) & (1095) \\
\hline & $\begin{array}{l}\tilde{f}_{b, J L N}^{\star}(\#) \\
\text { (\#) }\end{array}$ & 728 & 705 & 677 & 842 & 935 & 832 & 867 & 937 & 761 & 873 & 937 & 744 \\
\hline & & $(947)$ & (1006) & (926) & $(975)$ & (998) & $(855)$ & $(842)$ & $(880)$ & (859) & $(959)$ & (873) & $(864)$ \\
\hline
\end{tabular}

Table 2: Case B. The average ISEs $\times 10^{6}$.

The number in the parentheses stands for the standard deviation $\times 10^{6}$ of the ISEs.

\begin{tabular}{|c|c|c|c|c|c|c|c|c|c|c|c|c|c|}
\hline$n$ & linear & $A_{2}$ & $A_{1.5}$ & $A_{1}$ & $A_{0.5}$ & $A_{-0.5}$ & $A_{-1}$ & $A_{-1.5}$ & $A_{-2}$ & $I G$ & $B S$ & $R I G$ & $L N[-1 / 2]$ \\
\hline \multirow[t]{4}{*}{100} & $\widehat{f}_{b}^{(\#)}$ & 6452 & 6480 & 6049 & 5578 & 5924 & 6652 & 7505 & 7983 & 6565 & 7184 & 7466 & 6478 \\
\hline & & $(5871)$ & $(7440)$ & $(7483)$ & (7131) & (6366) & $(6154)$ & (6561) & $(6147)$ & $(7350)$ & $(8495)$ & (7694) & (7071) \\
\hline & $\widehat{f}_{b, J L N}^{(\#)}$ & 3168 & 3639 & 3899 & 4390 & 4548 & 4455 & 4543 & 5041 & 4088 & 4288 & 4010 & 3921 \\
\hline & & (4128) & (5740) & $(5521)$ & (6380) & $(5223)$ & $(4549)$ & $(3780)$ & $(4184)$ & $(4366)$ & (5226) & (4941) & (4401) \\
\hline \multirow[t]{4}{*}{300} & $\widehat{f}_{b}^{(\#)}$ & 2821 & 2634 & 2374 & 2113 & 2217 & 2628 & 3043 & 3309 & 2474 & 2756 & 3014 & 2474 \\
\hline & & (2599) & $(2804)$ & $(2787)$ & $(2642)$ & $(2062)$ & $(2245)$ & (2339) & (1980) & $(2352)$ & $(2752)$ & $(2828)$ & $(2368)$ \\
\hline & $\widehat{f}_{b, J L N}^{(\#)}$ & 1256 & 1296 & 1407 & 1519 & 1637 & 1696 & 1868 & 2052 & 1538 & 1562 & 1568 & 1493 \\
\hline & & (1475) & $(1524)$ & $(1752)$ & (1790) & $(1506)$ & (1388) & $(1464)$ & (1535) & (1502) & (1706) & (1764) & $(1476)$ \\
\hline$n$ & two-regime & $A_{2}$ & $A_{1.5}$ & $A_{1}$ & $A_{0.5}$ & $A_{-0.5}$ & $A_{-1}$ & $A_{-1.5}$ & $A_{-2}$ & $I G$ & $B S$ & $R I G$ & $L N[-1 / 2]$ \\
\hline \multirow[t]{4}{*}{100} & $\widetilde{f}_{b}^{\star}(\#)$ & 5346 & 5368 & 5242 & 5814 & 6140 & 5592 & 5688 & 5789 & 5551 & 5848 & 5783 & 5603 \\
\hline & & (6183) & $(7209)$ & $(6333)$ & $(7612)$ & $(6582)$ & $(5225)$ & $(4956)$ & $(4781)$ & $(6274)$ & (6198) & (5209) & $(6648)$ \\
\hline & $\begin{array}{l}\tilde{f}_{b, J L N}^{\star(\#)} \\
\text { (\#) }\end{array}$ & 4684 & 4592 & 4437 & 4429 & 4749 & 4869 & 5270 & 5505 & 4718 & 5658 & 6110 & 4845 \\
\hline & & $(4748)$ & $(5822)$ & $(5583)$ & $(6522)$ & $(5649)$ & $(4664)$ & $(4780)$ & $(4708)$ & (4868) & $(5101)$ & (5023) & (6106) \\
\hline \multirow[t]{4}{*}{300} & $\tilde{f}_{b}^{\star}(\#)$ & 2053 & 2043 & 1948 & 2106 & 2232 & 2128 & 2221 & 2324 & 2041 & 2179 & 2222 & 2028 \\
\hline & & $(2081)$ & $(2408)$ & (2038) & $(2515)$ & (1975) & $(1870)$ & (1966) & (1969) & (1961) & $(2056)$ & (1919) & (1968) \\
\hline & $\tilde{f}_{b, J L N}^{\star(\#)}$ & 1925 & 1774 & 1619 & 1521 & 1690 & 1909 & 2119 & 2288 & 1807 & 2182 & 2417 & 1817 \\
\hline & & (1899) & $(1710)$ & (1601) & (1685) & $(1752)$ & $(1777)$ & $(1742)$ & (1836) & (1777) & $(1825)$ & (1783) & (1840) \\
\hline
\end{tabular}


Table 3: Case C. The average ISEs $\times 10^{6}$.

The number in the parentheses stands for the standard deviation $\times 10^{6}$ of the ISEs.

\begin{tabular}{|c|c|c|c|c|c|c|c|c|c|c|c|c|c|}
\hline$n$ & linear & $A_{2}$ & $A_{1.5}$ & $A_{1}$ & $A_{0.5}$ & $A_{-0.5}$ & $A_{-1}$ & $A_{-1.5}$ & $A_{-2}$ & $I G$ & $B S$ & $R I G$ & $L N[-1 / 2]$ \\
\hline \multirow[t]{4}{*}{100} & $\widehat{f}_{b}^{(\#)}$ & 7178 & 6656 & 6045 & 5411 & 5763 & 6688 & 7454 & 8060 & 6462 & 6926 & 7545 & 6482 \\
\hline & & $(4902)$ & $(4722)$ & $(4604)$ & $(4325)$ & (4565) & (4967) & $(4944)$ & (4936) & $(4970)$ & $(5060)$ & (5310) & (4912) \\
\hline & $\widehat{f}_{b, J L N}^{(\#)}$ & 8002 & 7052 & 6077 & 5182 & 5840 & 8709 & 11444 & 13437 & 7415 & 7734 & 9594 & 7480 \\
\hline & & $(4845)$ & $(4818)$ & $(4563)$ & (4328) & (4469) & $(5297)$ & (6533) & (7794) & $(4959)$ & (5287) & $(5300)$ & (5011) \\
\hline \multirow[t]{4}{*}{300} & $\widehat{f}_{b}^{(\#)}$ & 3264 & 2969 & 2594 & 2234 & 2323 & 2787 & 3213 & 3577 & 2699 & 2985 & 3332 & 2703 \\
\hline & & (2000) & (1911) & $(1785)$ & (1697) & (1726) & $(1870)$ & (2049) & $(2178)$ & $(1844)$ & (1897) & $(2072)$ & (1852) \\
\hline & $\widehat{f}_{b, J L N}^{(\#)}$ & 3155 & 2757 & 2343 & 1941 & 2166 & 3920 & 7310 & 9415 & 2624 & 2877 & 3394 & 2622 \\
\hline & & $(2172)$ & (1963) & (1818) & $(1542)$ & $(1782)$ & $(3653)$ & $(5676)$ & $(6497)$ & $(2149)$ & $(2122)$ & $(2474)$ & $(2124)$ \\
\hline$n$ & two-regime & $A_{2}$ & $A_{1.5}$ & $A_{1}$ & $A_{0.5}$ & $A_{-0.5}$ & $A_{-1}$ & $A_{-1.5}$ & $A_{-2}$ & $I G$ & $B S$ & $R I G$ & $L N[-1 / 2]$ \\
\hline \multirow[t]{4}{*}{100} & $\tilde{f}_{b}^{\star}(\#)$ & 6131 & 6032 & 5826 & 5539 & 5853 & 6587 & 6914 & 7138 & 6243 & 6268 & 6386 & 6276 \\
\hline & & $(3885)$ & $(4154)$ & (4294) & (4308) & $(4244)$ & (4016) & $(3607)$ & $(3426)$ & $(4094)$ & $(3874)$ & $(3400)$ & (4027) \\
\hline & $\begin{array}{l}\tilde{f}_{b, J L N}^{\star(\#)} \\
\text { (\#) }\end{array}$ & 6132 & 5920 & 5735 & 5460 & 6054 & 6881 & 7212 & 7293 & 6359 & 6356 & 6389 & 6433 \\
\hline & & (3493) & $(3657)$ & $(3951)$ & $(4217)$ & (4218) & $(3473)$ & $(3284)$ & (2948) & $(3712)$ & $(3621)$ & $(3351)$ & $(3706)$ \\
\hline \multirow[t]{4}{*}{300} & $\tilde{f}_{b}^{\star(\#)}$ & 2743 & 2576 & 2379 & 2257 & 2351 & 2614 & 3219 & 3745 & 2485 & 2655 & 3008 & 2514 \\
\hline & & $(1729)$ & $(1716)$ & $(1716)$ & (1739) & $(1765)$ & (1853) & $(2056)$ & (1939) & $(1758)$ & $(1762)$ & (1801) & (1793) \\
\hline & $\begin{array}{l}\tilde{f}_{b, J L N}^{\star}(\#) \\
\text { (\#) }\end{array}$ & 3028 & 2756 & 2440 & 2050 & 2325 & 3162 & 4071 & 4619 & 2906 & 3173 & 3755 & 2952 \\
\hline & & (1712) & (1688) & (1676) & (1486) & (1697) & (1963) & (1891) & (1625) & $(1920)$ & (1593) & (1276) & (1990) \\
\hline
\end{tabular}

Table 4: Case D. The average ISEs $\times 10^{6}$.

The number in the parentheses stands for the standard deviation $\times 10^{6}$ of the ISEs.

\begin{tabular}{|c|c|c|c|c|c|c|c|c|c|c|c|c|c|}
\hline$n$ & linear & $A_{2}$ & $A_{1.5}$ & $A_{1}$ & $A_{0.5}$ & $A_{-0.5}$ & $A_{-1}$ & $A_{-1.5}$ & $A_{-2}$ & $I G$ & $B S$ & $R I G$ & $L N[-1 / 2]$ \\
\hline \multirow[t]{4}{*}{100} & $\widehat{f}_{b}^{(\#)}$ & 4665 & 4230 & 3669 & 3168 & 3155 & 3800 & 4600 & 5371 & 3708 & 4170 & 4705 & 3721 \\
\hline & & $(2851)$ & $(2744)$ & $(2491)$ & $(2295)$ & $(2316)$ & (2659) & (3011) & $(3326)$ & (2599) & $(2736)$ & (2907) & $(2586)$ \\
\hline & $\widehat{f}_{b, J L N}^{(\#)}$ & 4914 & 4269 & 3584 & 3227 & 3253 & 4441 & 5817 & 6725 & 3708 & 4177 & 5083 & 3838 \\
\hline & & (3030) & $(2843)$ & $(2587)$ & $(2344)$ & $(2493)$ & $(3470)$ & $(3855)$ & $(3956)$ & $(2794)$ & $(2955)$ & $(3523)$ & (2954) \\
\hline \multirow[t]{4}{*}{300} & $\widehat{f}_{b}^{(\#)}$ & 2124 & 1908 & 1677 & 1490 & 1476 & 1690 & 1949 & 2187 & 1662 & 1886 & 2098 & 1665 \\
\hline & & (1188) & (1108) & $(1022)$ & $(965)$ & (961) & (1051) & (1138) & (1244) & (1020) & (1113) & (1183) & (1022) \\
\hline & $\widehat{f}_{b, J L N}^{(\#)}$ & 2106 & 1828 & 1554 & 1467 & 1421 & 1648 & 2929 & 4104 & 1543 & 1772 & 2079 & 1562 \\
\hline & & (1270) & $(1144)$ & (1044) & (1023) & $(984)$ & $(1271)$ & $(2836)$ & $(3330)$ & (1050) & (1132) & (1272) & (1066) \\
\hline$n$ & two-regime & $A_{2}$ & $A_{1.5}$ & $A_{1}$ & $A_{0.5}$ & $A_{-0.5}$ & $A_{-1}$ & $A_{-1.5}$ & $A_{-2}$ & $I G$ & $B S$ & $R I G$ & $L N[-1 / 2]$ \\
\hline \multirow[t]{4}{*}{100} & $\widetilde{f}_{b}^{\star}(\#)$ & 4373 & 3882 & 3463 & 3208 & 3261 & 3865 & 5052 & 5618 & 3549 & 3976 & 5193 & 3662 \\
\hline & & $(2454)$ & $(2448)$ & $(2383)$ & $(2332)$ & $(2354)$ & $(2719)$ & $(2693)$ & $(2400)$ & $(2524)$ & $(2466)$ & $(2321)$ & $(2622)$ \\
\hline & $\begin{array}{l}\tilde{f}_{b, J L N}^{\star(\#)} \\
\text { (\#) }\end{array}$ & 5004 & 4699 & 4237 & 3875 & 3814 & 4597 & 5170 & 5507 & 4285 & 5594 & 5864 & 4471 \\
\hline & & (1951) & $(2123)$ & $(2414)$ & $(2432)$ & $(2375)$ & $(2595)$ & (2397) & $(2364)$ & $(2710)$ & (1689) & $(1560)$ & $(2741)$ \\
\hline \multirow[t]{4}{*}{300} & $\tilde{f}_{b}^{\star}(\#)$ & 1660 & 1586 & 1516 & 1484 & 1499 & 1538 & 1874 & 2897 & 1511 & 1590 & 1838 & 1509 \\
\hline & & (1033) & $(1026)$ & (1010) & $(987)$ & (1002) & (1013) & (1543) & $(2281)$ & (1005) & $(1076)$ & (1417) & (1010) \\
\hline & $\tilde{f}_{b, J L N}^{\star(\#)}$ & 1896 & 1724 & 1623 & 1580 & 1628 & 1741 & 2328 & 2966 & 1635 & 3160 & 4715 & 1637 \\
\hline & & (1186) & (1089) & (1085) & (1086) & (1073) & (1158) & (1659) & $(1850)$ & (1114) & $(2090)$ & (1375) & (1153) \\
\hline
\end{tabular}


Table 5: Case E. The average ISEs $\times 10^{6}$.

The number in the parentheses stands for the standard deviation $\times 10^{6}$ of the ISEs.

\begin{tabular}{cccccccccccccc}
\hline$n$ & linear & $A_{2}$ & $A_{1.5}$ & $A_{1}$ & $A_{0.5}$ & $A_{-0.5}$ & $A_{-1}$ & $A_{-1.5}$ & $A_{-2}$ & $I G$ & $B S$ & $R I G$ & $L N[-1 / 2]$ \\
\hline 100 & $\widehat{f}_{b}^{(\#)}$ & 18180 & 17507 & 16301 & 16554 & 17644 & 18206 & 20264 & 21747 & 17373 & 18362 & 19808 & 17458 \\
& & $(19993)$ & $(20660)$ & $(20420)$ & $(20261)$ & $(20074)$ & $(18769)$ & $(19102)$ & $(17432)$ & $(20823)$ & $(21422)$ & $(22564)$ & $(21431)$ \\
& $\widehat{f}_{b, J L N}^{(\#)}$ & 15038 & 15448 & 15117 & 16095 & 17172 & 17181 & 20203 & 28981 & 15994 & 16648 & 17890 & 15809 \\
& & $(15160)$ & $(19084)$ & $(18934)$ & $(17961)$ & $(16453)$ & $(13954)$ & $(15101)$ & $(26322)$ & $(14895)$ & $(18077)$ & $(18604)$ & $(14917)$ \\
300 & $\widehat{f}_{b}^{(\#)}$ & 7431 & 7027 & 6492 & 6565 & 7097 & 7403 & 8287 & 8966 & 6984 & 7361 & 7982 & 6961 \\
& & $(5803)$ & $(5940)$ & $(5727)$ & $(5919)$ & $(5937)$ & $(5527)$ & $(5801)$ & $(5579)$ & $(5824)$ & $(5826)$ & $(6148)$ & $(5830)$ \\
& $\widehat{f}_{b, J L N}^{(\#)}$ & 6287 & 6271 & 6085 & 6485 & 7235 & 6946 & 7813 & 11065 & 6472 & 6778 & 7246 & 6451 \\
& & $(5597)$ & $(6471)$ & $(6578)$ & $(6224)$ & $(5925)$ & $(5207)$ & $(6337)$ & $(11816)$ & $(5591)$ & $(6354)$ & $(6789)$ & $(5720)$ \\
\hline$n$ & two-regime & $A 2$ & $A_{1.5}$ & $A_{1}$ & $A_{0.5}$ & $A_{-0.5}$ & $A_{-1}$ & $A_{-1.5}$ & $A_{-2}$ & $I G$ & $B S$ & $R I G$ & $L N[-1 / 2]$ \\
\hline 100 & $\widetilde{f}_{b}^{(\#)}$ & 12778 & 13282 & 14334 & 16933 & 18391 & 15339 & 14276 & 14089 & 14853 & 14236 & 13404 & 15064 \\
& & $(18000)$ & $(18217)$ & $(18190)$ & $(18570)$ & $(18115)$ & $(12745)$ & $(12241)$ & $(12211)$ & $(15453)$ & $(15446)$ & $(16331)$ & $(17311)$ \\
& $\widetilde{f}_{b, J L N}^{(\#)}$ & 8689 & 9066 & 9955 & 13492 & 13781 & 11304 & 11146 & 11441 & 10955 & 11325 & 10642 & 10437 \\
& & $(13676)$ & $(13839)$ & $(11844)$ & $(12669)$ & $(12878)$ & $(10943)$ & $(10453)$ & $(10544)$ & $(11499)$ & $(12542)$ & $(10778)$ & $(11068)$ \\
300 & $\widetilde{f}_{b}^{(\#)}$ & 4993 & 5263 & 5840 & 6954 & 7584 & 6560 & 6069 & 5822 & 6297 & 5849 & 5511 & 6312 \\
& & $(5666)$ & $(5640)$ & $(5844)$ & $(6635)$ & $(6110)$ & $(5136)$ & $(4730)$ & $(4584)$ & $(5846)$ & $(5747)$ & $(5769)$ & $(6078)$ \\
& $\widetilde{f}_{b, J L N}^{(\#)}$ & 3229 & 3594 & 4303 & 6235 & 6623 & 4897 & 4519 & 4502 & 4627 & 4416 & 4211 & 4413 \\
& & $(3626)$ & $(4450)$ & $(4638)$ & $(5506)$ & $(5144)$ & $(4023)$ & $(3841)$ & $(3843)$ & $(4372)$ & $(4371)$ & $(3936)$ & $(4060)$ \\
\hline
\end{tabular}


Table 6: The average ISEs $\times 10^{6}$.

The number in the parentheses stands for the standard deviation $\times 10^{6}$ of the ISEs.

\begin{tabular}{|c|c|c|c|c|c|c|c|c|c|c|}
\hline & & linear & $A_{2}$ & $A_{1.5}$ & $A_{1}$ & $A_{0.5}$ & $A_{-0.5}$ & $A_{-1}$ & $A_{-1.5}$ & $A_{-2}$ \\
\hline \multirow{30}{*}{$n=100$} & \multirow[t]{6}{*}{ Case A } & \multirow{2}{*}{$\widehat{f}_{b, S S_{1}}^{(\#)}$} & 2289 & 2289 & 2211 & 2393 & 2504 & 2544 & 2963 & 3471 \\
\hline & & & $(2684)$ & $(3121)$ & $(2861)$ & $(2847)$ & $(2255)$ & $(2305)$ & $(2452)$ & (2733) \\
\hline & & \multirow[t]{2}{*}{$\widehat{f}_{b, S S_{1}}^{(\#),+}$} & 2288 & 2288 & 2221 & 2375 & 2504 & 2544 & 2963 & 3480 \\
\hline & & & $(2684)$ & (3121) & (2935) & $(2807)$ & $(2260)$ & $(2302)$ & $(2451)$ & $(2760)$ \\
\hline & & \multirow{2}{*}{$\widehat{f}_{b, J F_{1}}^{(\#)}$} & 2120 & 2022 & 2123 & 2353 & 2630 & 2414 & 2973 & 3674 \\
\hline & & & $(2487)$ & (2488) & $(2945)$ & $(2879)$ & (2393) & $(2463)$ & $(2682)$ & $(3054)$ \\
\hline & \multirow[t]{6}{*}{ Case B } & \multirow[t]{2}{*}{$\widehat{f}_{b, S S_{1}}^{(\#)}$} & 4088 & 4297 & 4368 & 4659 & 4673 & 4693 & 5334 & 5969 \\
\hline & & & (4789) & (5453) & (5791) & $(6653)$ & (5257) & (4184) & $(4556)$ & $(4762)$ \\
\hline & & \multirow[t]{2}{*}{$\widehat{f}_{b, S S_{1}}^{(\#),+}$} & 4087 & 4299 & 4371 & 4657 & 4672 & 4692 & 5334 & 6000 \\
\hline & & & (4789) & $(5460)$ & (5801) & (6643) & (5260) & (4184) & $(4557)$ & (4863) \\
\hline & & \multirow[t]{2}{*}{$\widehat{f}_{b, J F_{1}}^{(\#)}$} & 3458 & 3656 & 4023 & 4447 & 4700 & 4159 & 4815 & 5667 \\
\hline & & & (4123) & (4990) & (5803) & (6333) & $(5270)$ & $(4240)$ & $(4835)$ & (5169) \\
\hline & \multirow[t]{6}{*}{ Case C } & \multirow[t]{2}{*}{$\widehat{f}_{b, S S_{1}}^{(\#)}$} & 7796 & 7204 & 6295 & 5308 & 5847 & 7164 & 8343 & 9218 \\
\hline & & & $(4918)$ & $(4930)$ & $(4578)$ & (4407) & $(4453)$ & $(4503)$ & (4943) & $(5446)$ \\
\hline & & \multirow[t]{2}{*}{$\widehat{f}_{b, S S_{1}}^{(\#),+}$} & 7807 & 7223 & 6298 & 5318 & 5848 & 7163 & 8350 & 9216 \\
\hline & & & (4900) & (4929) & (4571) & (4415) & $(4454)$ & (4499) & (4940) & (5441) \\
\hline & & \multirow[t]{2}{*}{$\widehat{f}_{b, J F_{1}}^{(\#)}$} & 9043 & 8059 & 6805 & 5372 & 5720 & 6704 & 7870 & 8864 \\
\hline & & & $(4567)$ & $(5084)$ & $(4782)$ & $(4385)$ & $(4379)$ & $(4576)$ & $(5235)$ & (6167) \\
\hline & \multirow[t]{6}{*}{ Case D } & \multirow[t]{2}{*}{$\widehat{f}_{b, S S_{1}}^{(\#)}$} & 5195 & 4601 & 3841 & 3133 & 3047 & 3825 & 4617 & 5182 \\
\hline & & & $(2818)$ & (2964) & $(2719)$ & (2333) & $(2384)$ & (2911) & (3037) & $(3100)$ \\
\hline & & \multirow[t]{2}{*}{$\widehat{f}_{b, S S_{1}}^{(\#),+}$} & 5192 & 4603 & 3831 & 3120 & 3046 & 3822 & 4608 & 5153 \\
\hline & & & $(2825)$ & $(2982)$ & $(2711)$ & (2301) & (2388) & (2911) & (3037) & (3097) \\
\hline & & \multirow[t]{2}{*}{$\widehat{f}_{b, J F_{1}}^{(\#)}$} & 5734 & 5099 & 4175 & 3275 & 3174 & 3933 & 4665 & 5334 \\
\hline & & & (2956) & (3097) & (2793) & $(2355)$ & $(2426)$ & $(2883)$ & $(2985)$ & $(3206)$ \\
\hline & \multirow[t]{6}{*}{ Case E } & \multirow[t]{2}{*}{$\widehat{f}_{b, S S_{1}}^{(\#)}$} & 11855 & 12568 & 13174 & 14023 & 14789 & 13752 & 14890 & 17189 \\
\hline & & & $(12877)$ & (18628) & $(20412)$ & (18530) & (15457) & (12444) & (10726) & (11400) \\
\hline & & \multirow[t]{2}{*}{$\widehat{f}_{b, S S_{1}}^{(\#),+}$} & 11866 & 12563 & 13174 & 13995 & 14779 & 13763 & 14890 & 17189 \\
\hline & & & (13002) & (18599) & (20359) & $(18571)$ & $(15464)$ & (12603) & $(10726)$ & (11400) \\
\hline & & \multirow[t]{2}{*}{$\widehat{f}_{b, J F_{1}}^{(\#)}$} & 12321 & 12416 & 12837 & 14622 & 14962 & 12936 & 14003 & 18814 \\
\hline & & & $(12239)$ & $(15400)$ & $(18734)$ & (18552) & (14210) & $(8751)$ & (12315) & (18493) \\
\hline
\end{tabular}


Table 6: Continued.

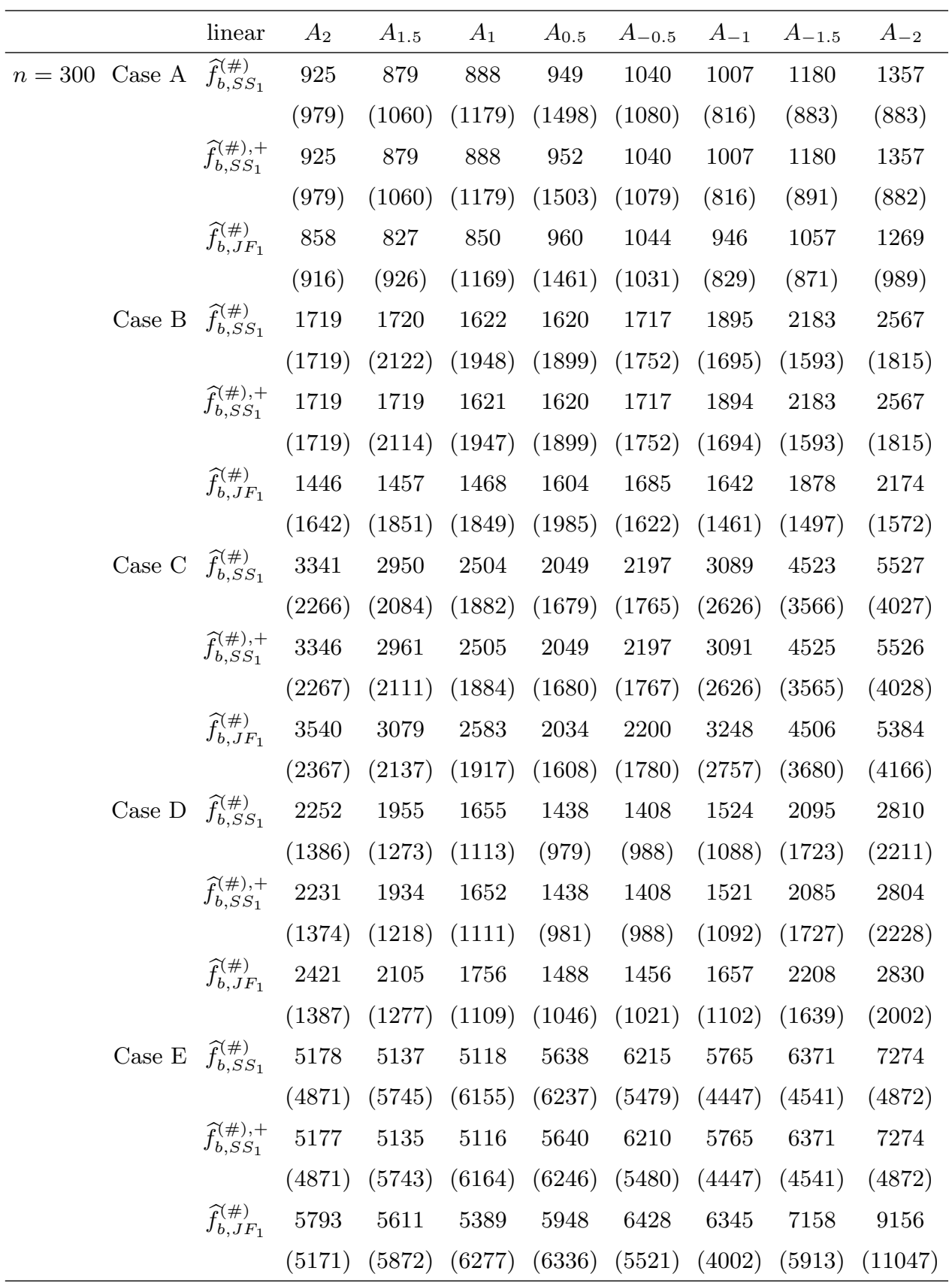

